Article

\title{
Inverse Problems with Unknown Boundary Conditions and Final Overdetermination for Time Fractional Diffusion-Wave Equations in Cylindrical Domains
}

\author{
Jaan Janno (1)
}

Citation: Janno, J. Inverse Problems with Unknown Boundary Conditions and Final Overdetermination for Time Fractional Diffusion-Wave Equations in Cylindrical Domains. Mathematics 2021, 9, 2541. https:// doi.org/10.3390/math9202541

Academic Editor: Christine Böckmann

Received: 7 September 2021 Accepted: 6 October 2021

Published: 10 October 2021

Publisher's Note: MDPI stays neutral with regard to jurisdictional claims in published maps and institutional affiliations.

Copyright: (C) 2021 by the author. Licensee MDPI, Basel, Switzerland. This article is an open access article distributed under the terms and conditions of the Creative Commons Attribution (CC BY) license (https:/ / creativecommons.org/licenses/by/ $4.0 /)$.
Department of Cybernetics, Tallinn University of Technology, Ehitajate tee 5, 19086 Tallinn, Estonia; jaan.janno@ttu.ee

\begin{abstract}
Inverse problems to reconstruct a solution of a time fractional diffusion-wave equation in a cylindrical domain are studied. The equation is complemented by initial and final conditions and partly given boundary conditions. Two cases are considered: (1) full boundary data on a lateral hypersurface of the cylinder are given, but the boundary data on bases of the cylinder are specified in a neighborhood of a final time; (2) boundary data on the whole boundary of the cylinder are specified in a neighborhood of the final time, but the cylinder is either a cube or a circular cylinder. Uniqueness of solutions of the inverse problems is proved. Uniqueness for similar problems in an interval and a disk is established, too.
\end{abstract}

Keywords: inverse problem; fractional diffusion-wave equation; final overdetermination; unknown boundary condition

\section{Introduction}

Theoretical study of fractional differential equations that generalize mathematical models of various anomalous processes have been a focus of mathematicians in the last few decades. An example is the time fractional diffusion-wave equation $[1,2]$ that contains a fractional time derivative of the order $\alpha \in(0,1) \cup(1,2)$ and comprises equations governing subdiffusion $(\alpha \in(0,1))$ [3-5] and fractional wave $(\alpha \in(1,2))$ [6-8] processes. The usual parabolic equation with time derivative of the integer order $\alpha=1$ lies between these two fractional cases.

Often, parameters of equations are a priori unknown. A common way to determine them is to solve inverse problems that use measurements of states of the processes. Depending on the practical situation, the state can be measured at interior or boundary points of a medium over the time [2,9-12] or at fixed time moments over the space, e.g., at a final time [13-16].

Inverse problems to reconstruct space-dependent parameters of fractional diffusion equations by means of final measurements have been studied in several papers. Many methods that are applicable to such problems in the usual integer case, such as the Fredholm alternative [15], extremum principles [17], Fourier method [2,16,18-20], and Carleman estimates [21], can be successfully adjusted to the fractional case.

Recently, the author of the present paper proved that a time-dependent factor $f(t)$ of a source term in the fractional diffusion-wave equation is uniquely recovered by the final data [22]. A character of this problem in the fractional case essentially differs from that one in the integer case. The key feature is a behavior at $+\infty$ of a kernel contained in formula of a Fourier coefficient of a state function. In the case $\alpha=1$, this kernel is exponentially decaying, but, in a fractional case $\alpha \in(0,1) \cup(1,2)$, it admits a power-type asymptotic expansion. Assuming that $f(t)$ is a priori known in a left neighborhood $(T-\delta, T)$ of the final moment $T$, the aforementioned power-type expansion enables reduction of the 
inverse problem to a moment problem and, in turn, establishment of the uniqueness of $f(t)$ for $t \in(0, T-\delta)$.

In this paper, we continue the study of inverse problems with time-dependent unknowns and over-specified final data. We consider two problems for the time fractional diffusion-wave equation in a cylindrical domain. In both problems, the initial and final conditions are given. In the first problem, the boundary conditions are prescribed for $t \in(0, T)$ on the lateral surface and for $t \in(T-\delta, T)$ on both bases. In the second problem, the boundary conditions are prescribed for $t \in(T-\delta, T)$ on the whole boundary, and the domain is either a cube or a circular cylinder.

From the physical viewpoint, such problems may arise when the regime of energy exchange between the domain and a surrounding medium is partly or fully unknown before the time value $T-\delta$.

We will prove that solutions of the mentioned problems are unique. As corollaries, we establish uniqueness for similar inverse problems on an interval and a disk, too. At the end of the paper, we briefly discuss some other questions: ill-posedness, open problems, etc.

Problems with final overdetermination and unknown boundary conditions are completely new and have not yet been studied neither in the fractional nor in the classical integer case.

\section{Formulation of Problems}

Let $\omega \subset \mathbb{R}^{d}, d \geq 1$, be an open bounded domain with the boundary $\partial \omega$ and $\Omega=\omega \times(0,1)$. We use the following notation for points of $\Omega$ and their coordinates:

$$
(x, y) \in \Omega, x=\left(x_{1}, \ldots, x_{d}\right) \in \omega, y \in(0,1),
$$

and the following abbreviated notation for partial derivatives:

$$
\begin{aligned}
& \partial_{x_{i}}^{m} f(t, x, y)=\frac{\partial^{m}}{\partial x_{i}^{m}} f(t, x, y), \partial_{y}^{m} f(t, x, y)=\frac{\partial^{m}}{\partial y^{m}} f(t, x, y), \\
& \partial_{t}^{m} f(t, x, y)=\frac{\partial^{m}}{\partial t^{m}} f(t, x, y), \quad m \in \mathbb{N} .
\end{aligned}
$$

In case $m=1$, we omit the superscript $m$. Moreover, we denote the Laplace operators in $\Omega$ and $\omega$ as follows:

$$
\Delta=\Delta_{d}+\partial_{y}^{2} \text { and } \Delta_{d}=\sum_{i=1}^{d} \partial_{x_{i}}^{2} .
$$

Let $\alpha \in(0,1) \cup(1,2)$. Let $\lfloor\cdot\rfloor$ stand for the floor function and $q \in \mathbb{R}$. We consider the following time fractional diffusion-wave equation in the domain $\Omega$ (cf. References [1,22]):

$$
\begin{aligned}
& D_{t}^{\alpha-\lfloor\alpha\rfloor}\left[\partial_{t}^{\lfloor\alpha\rfloor} u-\left.\partial_{t}^{\lfloor\alpha\rfloor} u\right|_{t=0}\right](t, x, y)-(\Delta-q) u(t, x, y)=F(t, x, y), \\
& (x, y) \in \Omega, t>0 .
\end{aligned}
$$

Here, the symbol $D_{t}^{\beta}$ stands for the Riemann-Liouville fractional derivative of the order $\beta$ with respect to $t$ that is defined as follows:

$$
D_{t}^{\beta} f(t)=\frac{d}{d t} \int_{0}^{t} \frac{(t-\tau)^{-\beta}}{\Gamma(1-\beta)} f(\tau) d \tau, \quad t>0, \quad 0<\beta<1 .
$$

We mention that the term $D_{t}^{\alpha-\lfloor\alpha\rfloor}\left[\partial_{t}^{\lfloor\alpha\rfloor} u-\left.\partial_{t}^{\lfloor\alpha\rfloor} u\right|_{t=0}\right]$ is the Caputo fractional derivative of the order $\alpha$ of $u$. Namely, in case $u$ is $\lfloor\alpha\rfloor+1$ times differentiable with respect to $t$, this can be rewritten in the following form:

$$
D_{t}^{\alpha-\lfloor\alpha\rfloor}\left[\partial_{t}^{\lfloor\alpha\rfloor} u-\left.\partial_{t}^{\lfloor\alpha\rfloor} u\right|_{t=0}\right](t, x, y)=\int_{0}^{t} \frac{(t-\tau)^{\lfloor\alpha\rfloor-\alpha}}{\Gamma(1+\lfloor\alpha\rfloor-\alpha)} \partial_{\tau}^{\lfloor\alpha\rfloor+1} u(\tau, x, y) d \tau
$$


where the right-hand side contains the Caputo derivative in the usual form.

Let $T>0$. We formulate the following (direct) problem for the function $u$ :

$$
\begin{aligned}
& D_{t}^{\alpha-\lfloor\alpha\rfloor}\left[\partial_{t}^{\lfloor\alpha\rfloor} u-\varphi_{\lfloor\alpha\rfloor}\right](t, x, y)-(\Delta-q) u(t, x, y)=F(t, x, y), \\
& \quad t \in(0, T), \quad(x, y) \in \Omega, \\
& \left.\partial_{t}^{j} u(t, x, y)\right|_{t=0}=\varphi_{j}(x, y), \quad(x, y) \in \Omega, \quad j \in\{0 ;\lfloor\alpha\rfloor\}, \\
& B u(t, x, y)=h(t, x, y), \quad t \in(0, T), x \in \partial \omega, y \in(0,1), \\
& \left.B_{0} u(t, x, y)\right|_{y=0}=g_{0}(t, x),\left.\quad B_{1} u(t, x, y)\right|_{y=1}=g_{1}(t, x), \quad t \in(0, T), x \in \omega .
\end{aligned}
$$

Here, $F, \varphi_{j}, j \in\{0 ;\lfloor\alpha\rfloor\}, h, g_{0}, g_{1}$ are given functions, and the boundary operators $B, B_{0}, B_{1}$ are defined as follows:

$$
\text { either } B=I \text { or } B=\partial_{v}+\gamma I, \gamma \geq 0 \text {, }
$$

where $I$ is the unity operator, $v=v(x)$ is the outer normal vector of $\partial \omega$ at $x \in \partial \omega$, $\partial_{v}=\sum_{i=1}^{d} v_{i} \partial_{x_{i}}$, and

$$
\begin{aligned}
& \text { either } B_{0}=I, \quad B_{1}=I \\
& \text { or } B_{0}=I, \quad B_{1}=\partial_{y}+\gamma_{1} I, \quad \gamma_{1} \geq 0 \\
& \text { or } B_{0}=\partial_{y}-\gamma_{0} I, \quad B_{1}=\partial_{y}+\gamma_{1} I, \quad \gamma_{0} \geq 0, \gamma_{1} \geq 0 .
\end{aligned}
$$

Now, let us proceed to inverse problems to reconstruct the function $u$ in case of partly given boundary data. Suppose that either the pair $g_{0}, g_{1}$ or the triplet $g_{0}, g_{1}, h$ of boundary functions is given only in the time subinterval $t \in(T-\delta, T)$, where $\delta$ is some number between 0 and $T$. In order to recover $u$, we assume the following final condition:

$$
u(T, x, y)=\psi(x, y), \quad(x, y) \in \Omega,
$$

where $\psi$ is a given function.

The corresponding inverse problems are as follows.

IP1. Find $u$ that satisfies (1), (2), (3), (5) and

$$
\left.B_{0} u(t, x, y)\right|_{y=0}=g_{0}(t, x),\left.B_{1} u(t, x, y)\right|_{y=1}=g_{1}(t, x), \quad t \in(T-\delta, T), x \in \omega .
$$

IP2. Find $u$ that satisfies (1), (2), (5) and

$$
\begin{aligned}
& B u(t, x, y)=h(t, x, y), \quad t \in(T-\delta, T), x \in \partial \omega, y \in(0,1), \\
& \left.B_{0} u(t, x, y)\right|_{y=0}=g_{0}(t, x),\left.B_{1} u(t, x, y)\right|_{y=1}=g_{1}(t, x), \quad t \in(T-\delta, T), x \in \omega .
\end{aligned}
$$

We will prove the uniqueness of solutions of IP1 and IP2 in Sections 4 and 5, respectively (Theorems 1-3).

A method that we use in the analysis of IP2 requires additional restrictions on the domain $\Omega$. We will will study this problem in special cases when $\Omega$ is either a cube or a circular cylinder.

We will also extend the uniqueness results to inverse problems on an interval and a disk (Corollaries 1 and 2). Formulations of the latter problems are given right before the mentioned corollaries.

\section{Preliminaries}

\subsection{Eigenvalues and Eigenfunctions}

Let $\left.\left(\mu_{k}, v_{k}\right)\right|_{k \in \mathcal{K}}$ be the set of solutions of the eigenvalue problem

$$
-\left(\Delta_{d}-q\right) v(x)=\mu v(x), x \in \omega,\left.B v\right|_{\partial \omega}=0,
$$


where $v_{k}, k \in \mathcal{K}$, form a complete orthogonal system in $L_{2}(\omega)$. We assume that the domain $\omega$ is sufficiently regular to guarantee $v_{k} \in H^{2}(\omega), k \in \mathcal{K}$. For instance, this is the case if $\partial \omega \in C^{2}$ (Reference [23], Thm. 3.10). Another example of such an $\omega$ is the hyperrectangle $\omega=\prod_{j=1}^{d}\left(0, a_{j}\right), a_{j}>0$.

Although $\mathcal{K}$ is countable, and it is possible to identify it with $\mathbb{N}$, in some cases below, it is more convenient to use other forms of $\mathcal{K}$.

Further, let $\left.\left(\varrho_{l}, w_{l}\right)\right|_{l \in \mathbb{N}}$ be the solutions of the one-dimensional eigenvalue problem

$$
-w^{\prime \prime}(y)=\varrho w(y), y \in(0,1), B_{0} w(0)=B_{1} w(1)=0,
$$

where $w_{l}, l \in \mathbb{N}$, form a complete orthogonal system in $L_{2}(0,1)$. We can specify $\varrho_{l}$ and $w_{l}$ as follows:

$$
\left.\begin{array}{l}
\varrho_{l}=(l \pi)^{2}, w_{l}(y)=\sin (l \pi y) \quad \text { in case } B_{0}=I, B_{1}=I, \\
\varrho_{l}=p_{l}^{2}, w_{l}(y)=\sin \left(p_{l} y\right) \quad \text { in case } B_{0}=I, B_{1}=\partial_{y}+\gamma_{1} I, \\
\text { where } p_{l} \text { are positive solutions of } p \cos p=-\gamma_{1} \sin p ; \\
\text { it holds that } p_{l} \in((l-1) \pi, l \pi), \\
\varrho_{l}=p_{l}^{2}, w_{l}(y)=\cos \left(p_{l} y\right)+\gamma_{0} y \operatorname{sinc}\left(p_{l} y\right) \\
\begin{array}{l}
\text { in case } B_{0}=\partial_{y}-\gamma_{0} I, B_{1}=\partial_{y}+\gamma_{1} I, \\
\text { where } p_{l} \text { are the nonnegative solutions of } \\
\left(\gamma_{0}+\gamma_{1}\right) p \cos p=\left(p^{2}-\gamma_{0} \gamma_{1}\right) \sin p ;
\end{array} \\
\text { it holds that } p_{l} \begin{cases}\in((l-1) \pi, l \pi) & \text { for } \gamma_{0}+\gamma_{1}>0 \\
=(l-1) \pi & \text { for } \gamma_{0}=\gamma_{1}=0 .\end{cases}
\end{array}\right\} .
$$

We do not need to normalize the eigenfunctions $v_{k}$ and $w_{l}$.

The quantities $\lambda_{k, l}=\mu_{k}+\varrho_{l}$ and $\mathrm{v}_{k, l}(x, y)=v_{k}(x) w_{l}(y), k \in \mathcal{K}, l \in \mathbb{N}$, satisfy the eigenvalue problem

$$
\begin{aligned}
& -(\Delta-q) \mathrm{v}(x, y)=\lambda \mathrm{v}(x, y),(x, y) \in \Omega, \\
& \left.B \mathrm{v}\right|_{\partial \omega \times(0,1)}=0,\left.B_{0} \mathrm{v}\right|_{\omega \times\{0\}}=\left.B_{1} \mathrm{v}\right|_{\omega \times\{1\}}=0 .
\end{aligned}
$$

The system $\mathrm{v}_{k, l}, k \in \mathcal{K}, l \in \mathbb{N}$, is complete in $L_{2}(\Omega)$.

\subsection{Abstract Functional Spaces}

Let $X$ be a complex Banach space and $G \subseteq \mathbb{R}$. As usual, $L_{p}(G ; X), p \in[1, \infty]$ denotes the abstract Lebesgue space, i.e.,

$$
\begin{aligned}
& L_{p}(G ; X)=\left\{f: G \mapsto X: \int_{G}\|f(t)\|_{X}^{p} d t<\infty\right\}, \quad 1 \leq p<\infty, \\
& L_{\infty}(G ; X)=\left\{f: G \mapsto X: \text { ess } \sup _{t \in G}\|f(t)\|_{X}<\infty\right\} .
\end{aligned}
$$

The space $C(G ; X)$ consists of functions that are continuous on $G$ with values in $X$ and

$$
C^{n}(G ; X)=\left\{f: G \mapsto X: f^{(i)} \in C(G ; X), i=0, \ldots, n\right\}, \quad n \in \mathbb{N} .
$$

In addition, we introduce the spaces

$$
H_{p}^{s}((0, T) ; X)=\left\{\left.f\right|_{(0, T)}: f \in H_{p}^{s}(\mathbb{R} ; X)\right\}, p \in(1, \infty), s>0,
$$

where

$$
H_{p}^{s}(\mathbb{R} ; X)=\left\{f \in L_{p}(\mathbb{R} ; X): \mathcal{F}^{-1}|\xi|^{s} \mathcal{F} f \in L_{p}(\mathbb{R} ; X)\right\},
$$


and $\mathcal{F}$ denotes the Fourier transform with the argument $\xi$. Moreover, we define the following space:

$$
{ }_{0} H_{p}^{s}((0, T) ; X)=\left\{\left.f\right|_{(0, T)}: f \in H_{p}^{s}(\mathbb{R} ; X) \text {, supp } f \subseteq[0, \infty)\right\} .
$$

In case $X$ is the set of complex numbers, we omit it in the notations of $H_{p}^{s}$ and ${ }_{0} H_{p}^{s}$. Next, we formulate a lemma that describes the domain of the operator $D_{t}^{\beta}$ in $L_{p}$.

Lemma 1. Let $X$ be a complex Hilbert space, $\beta \in(0,1)$ and $p \in(1, \infty)$. The operator $D_{t}^{\beta}$ is a bijection from ${ }_{0} H_{p}^{\beta}((0, T) ; X)$ to $L_{p}((0, T) ; X)$ and its inverse is $\frac{t^{\beta-1}}{\Gamma(\beta)} *$. (The function $\frac{t^{\beta-1}}{\Gamma(\beta)} * f$ is called the Riemann-Liouville fractional integral of the order $\beta$ of a function $f$ ).

Here, $*$ denotes the time convolution, i.e.,

$$
f_{1} * f_{2}(t)=\int_{0}^{t} f_{1}(t-\tau) f_{2}(\tau) d \tau
$$

Lemma 1 follows from Corollary 2.8.1 and discussions on p. 29 of Reference [24].

\subsection{Formula for Fourier Coefficients of Solution of Direct Problem}

Lemma 2. Let $\alpha \in(0,1) \cup(1,2), f \in C^{\lfloor\alpha\rfloor}[0, T], f^{(j)}(0)=0, j \in\{0 ;\lfloor\alpha\rfloor\}$ and $f^{(\lfloor\alpha\rfloor)} \in$ ${ }_{0} H_{p}^{\alpha-\lfloor\alpha\rfloor}(0, T)$ for some $p>1$. Then,

$$
f(t)=e_{\alpha}(-\lambda ; t) *\left(D_{t}^{\alpha-\lfloor\alpha\rfloor} f^{(\lfloor\alpha\rfloor)}(t)+\lambda f(t)\right), \quad t \in[0, T], \lambda \in \mathbb{R},
$$

where $e_{\alpha}$ is the $\alpha$-exponential function that is defined via the two-parametric Mittag-Leffler function $E_{\alpha, \alpha}$ as follows:

$$
e_{\alpha}(\lambda ; t)=t^{\alpha-1} E_{\alpha, \alpha}\left(\lambda t^{\alpha}\right)
$$

Formula (11) is deduced, e.g., in Example 42.2 of Reference [25]. It can also be derived by means of some relations available for Mittag-Leffler functions. Let us show this. Let us denote the right-hand side of (11) by RHS. Bringing the operator $\frac{d}{d t}$ from the term $D_{t}^{\alpha-\lfloor\alpha\rfloor}=\frac{d}{d t}\left[\frac{t^{-\gamma}}{\Gamma(1-\gamma)} *\right]$ in front of the convolution, using the formula $e_{\alpha}(-\lambda ; t) * \frac{t^{-\gamma}}{\Gamma(1-\gamma)}=$ $t^{\alpha-\gamma} E_{\alpha, \alpha-\gamma+1}\left(-\lambda t^{\alpha}\right)$ (see Reference [26], p. 61) with $\gamma=\alpha-\lfloor\alpha\rfloor$ and integrating by parts in case $\lfloor\alpha\rfloor=1$, we have RHS $=\frac{d}{d t}\left[\frac{d^{\lfloor\alpha\rfloor}}{d t^{\lfloor\alpha\rfloor}}\left(t^{\lfloor\alpha\rfloor} E_{\alpha,\lfloor\alpha\rfloor+1}\left(-\lambda t^{\alpha}\right)\right) * f(t)\right]+\lambda e_{\alpha}(-\lambda ; t) *$ $f(t)$. Since $\frac{d}{d t}\left(t E_{\alpha, 2}\left(-\lambda t^{\alpha}\right)\right)=E_{\alpha, 1}\left(-\lambda t^{\alpha}\right)$ (this can be verified by means of the series representation $\left.E_{\alpha, \beta}(z)=\sum_{n=0}^{\infty} \frac{z^{n}}{\Gamma(n \alpha+\beta)}\right)$, we further have RHS $=\frac{d}{d t}\left[E_{\alpha, 1}\left(-\lambda t^{\alpha}\right) * f(t)\right]+$ $\lambda e_{\alpha}(-\lambda ; t) * f(t)$. Finally, using the relations $E_{\alpha, 1}(0)=1$ and $\frac{d}{d t} E_{\alpha, 1}\left(-\lambda t^{\alpha}\right)=-\lambda e_{\alpha}(-\lambda ; t)$, we obtain RHS $=f(t)$.

We will deal with solutions of the direct problem that belong to the following space:

$$
\begin{aligned}
& \mathcal{U}_{\alpha}=\left\{u \in C^{\lfloor\alpha\rfloor}\left([0, T] ; L_{2}(\Omega)\right): \exists p \in(1, \infty):\right. \\
& \left.u \in L_{p}\left((0, T) ; H^{2}(\Omega)\right), \partial_{t}^{\lfloor\alpha\rfloor} u-\left.\partial_{t}^{\lfloor\alpha\rfloor} u\right|_{t=0} \in{ }_{0} H_{p}^{\alpha-\lfloor\alpha\rfloor}\left((0, T) ; L_{2}(\Omega)\right)\right\} .
\end{aligned}
$$

Then, the fractional derivative and Laplacian of $u$ in the Equation (1) are Lebesgue integrable functions, and $u$ has regular traces $\left.u\right|_{t=0},\left.B u\right|_{x \in \partial \omega},\left.B_{0} u\right|_{y=0},\left.B_{1} u\right|_{y=1}$. Moreover, due to well-known trace theorems, $u \in \mathcal{U}_{\alpha}$ implies that the data of the direct problem necessarily satisfy the conditions

$$
\begin{aligned}
& F \in L_{p}\left((0, T) ; L_{2}(\Omega)\right), \varphi_{j} \in L_{2}(\Omega), j \in\{0 ;\lfloor\alpha\rfloor\}, \\
& h \in L_{p}\left((0, T) ; L_{2}(\partial \omega \times(0,1))\right), \quad g_{0}, g_{1} \in L_{p}\left((0, T) ; L_{2}(\omega)\right) .
\end{aligned}
$$


In the next proposition, we deduce a formula for Fourier coefficients of the solution of (1)-(4) in the case the equation and initial conditions are homogeneous.

Proposition 1. Let $\alpha \in(0,1) \cup(1,2)$. Let a function $u$ belong to the space $\mathcal{U}_{\alpha}$ and solve $(1)-(4)$ with $F=0$ and $\varphi_{j}=0, j \in\{0 ;\lfloor\alpha\rfloor\}$. Then,

$$
\begin{aligned}
& u_{k, l}(t)=e_{\alpha}\left(-\lambda_{k, l} ; t\right) *\left((-1)^{\vartheta} \int_{\partial \omega} \partial_{v}^{\vartheta} v_{k}(x) h_{l}(t, x) d S\right. \\
& \left.+(-1)^{\vartheta_{0}+1} w_{l}^{\left(\vartheta_{0}\right)}(0) g_{0 ; k}(t)+(-1)^{\vartheta_{1}} w_{l}^{\left(\vartheta_{1}\right)}(1) g_{1 ; k}(t)\right), \quad t \in[0, T], \\
& k \in \mathcal{K}, l \in \mathbb{N},
\end{aligned}
$$

where

$$
\begin{aligned}
& u_{k, l}(t)=\left\langle u(t, \cdot, \cdot), \mathrm{v}_{k, l}\right\rangle_{L_{2}(\Omega)}, k \in \mathcal{K}, l \in \mathbb{N}, \\
& h_{l}(t, x)=\left\langle h(t, x, \cdot), w_{l}\right\rangle_{L_{2}(0,1)}, l \in \mathbb{N}, \\
& g_{0 ; k}(t)=\left\langle g_{0}(t, \cdot), v_{k}\right\rangle_{L_{2}(\omega)}, g_{1 ; k}(t)=\left\langle g_{1}(t, \cdot), v_{k}\right\rangle_{L_{2}(\omega)}, k \in \mathcal{K},
\end{aligned}
$$

$\langle\cdot, \cdot\rangle_{X}$ denotes the inner product in a Hilbert space $X, d S$ is the element of $\partial \omega$,

$$
\vartheta=\left\{\begin{array}{l}
1 \quad \text { in case } B=I \\
0 \quad \text { in case } B=\partial_{v}+\gamma I
\end{array}\right.
$$

and

$$
\left(\vartheta_{0}, \vartheta_{1}\right)= \begin{cases}(1,1) & \text { in case } B_{0}=I, B_{1}=I \\ (1,0) & \text { in case } B_{0}=I, B_{1}=\partial_{y}+\gamma_{1} I \\ (0,0) & \text { in case } B_{0}=\partial_{y}-\gamma_{0} I, B_{1}=\partial_{y}+\gamma_{1} I\end{cases}
$$

Proof. Due to the assumption $u \in \mathcal{U}_{\alpha}$ and (14), we have $h_{l} \in L_{p}\left((0, T) ; L_{2}(\partial \omega)\right), g_{0 ; k}, g_{1 ; k} \in$ $L_{p}(0, T)$. Therefore, the right-hand side of the Formula (15) is well-defined. Moreover, the functions $u_{k, l}$ belong to $C^{\lfloor\alpha\rfloor}[0, T]$ and satisfy $u_{k, l}^{(\lfloor\alpha\rfloor)} \in{ }_{0} H_{p}^{\alpha-\lfloor\alpha\rfloor}(0, T)$. Taking the inner product of the Equation (1) with $\mathrm{v}_{k, l}$ in $L_{2}(\Omega), k, l \in \mathbb{N}$, in case $F=0$, we obtain

$$
D_{t}^{\alpha-\lfloor\alpha\rfloor} u_{k, l}^{(\lfloor\alpha\rfloor)}(t)-\left\langle(\Delta-q) u(t, \cdot, \cdot), \mathrm{v}_{k, l}\right\rangle_{L_{2}(\Omega)}=0, t \in(0, T) .
$$

This, in view of $\Delta=\Delta_{d}+\partial_{y}^{2}$ and $\lambda_{k, l}=\mu_{k}+\varrho_{l}$, transforms to

$$
\begin{aligned}
& D_{t}^{\alpha-\lfloor\alpha\rfloor} u_{k, l}^{(\lfloor\alpha\rfloor)}(t)+\lambda_{k, l} u_{k, l}(t)=\left\langle\left(\mu_{k}+\Delta_{d}-q\right) u(t, \cdot, \cdot), \mathrm{v}_{k, l}\right\rangle_{L_{2}(\Omega)} \\
& +\left\langle\left(\varrho_{l}+\partial_{y}^{2}\right) u(t, \cdot, \cdot), \mathrm{v}_{k, l}\right\rangle_{L_{2}(\Omega)}, t \in(0, T) .
\end{aligned}
$$

Moreover, the assumption $\varphi_{j}=0, j \in\{0 ;\lfloor\alpha\rfloor\}$ implies $u_{k, l}^{(j)}(0)=0, j \in\{0 ;\lfloor\alpha\rfloor\}$. Thus, by means of Lemma 2, from (17), we obtain

$$
\begin{aligned}
& u_{k, l}(t)=e_{\alpha}\left(-\lambda_{k, l} ; t\right) *\left[\left\langle\left(\mu_{k}+\Delta_{d}-q\right) u(t, \cdot, \cdot), \mathrm{v}_{k, l}\right\rangle_{L_{2}(\Omega)}\right. \\
& \left.+\left\langle\left(\varrho_{l}+\partial_{y}^{2}\right) u(t, \cdot, \cdot), \mathrm{v}_{k, l}\right\rangle_{L_{2}(\Omega)}\right], \quad t \in[0, T] .
\end{aligned}
$$

In view of the Green's second identity and the relation $\left(\mu_{k}+\Delta_{d}-q\right) v_{k}=0$, we have

$$
\begin{aligned}
& \left\langle\left(\mu_{k}+\Delta_{d}-q\right) u, \mathrm{v}_{k, l}\right\rangle_{L_{2}(\Omega)}=\left\langle\left(\mu_{k}+\Delta_{d}-q\right) u, v_{k} w_{l}\right\rangle_{L_{2}(\Omega)} \\
& =Q+\left\langle u,\left(\mu_{k}+\Delta_{d}-q\right) v_{k} w_{l}\right\rangle_{L_{2}(\Omega)}=Q, \quad \text { where } \\
& Q=\int_{\partial \omega} v_{k}(x) \int_{0}^{1} \partial_{\nu} u(t, x, y) w_{l}(y) d y d S-\int_{\partial \omega} \partial_{\nu} v_{k}(x) \int_{0}^{1} u(t, x, y) w_{l}(y) d y d S .
\end{aligned}
$$


Using the boundary conditions $\left.B u\right|_{(0, T) \times \partial \omega \times(0,1)}=h$ and $\left.B v_{k}\right|_{\partial \omega}=0$, we obtain

$$
Q=-\int_{\partial \omega} \partial_{\nu} v_{k}(x) h_{l}(t, x) d S \quad \text { in case } B=I
$$

and

$$
\begin{aligned}
& Q=\int_{\partial \omega} v_{k}(x) \int_{0}^{1}\left(\partial_{v}+\gamma I\right) u(t, x, y) w_{l}(y) d y d S \\
& -\int_{\partial \omega}\left(\partial_{v}+\gamma I\right) v_{k}(x) \int_{0}^{1} u(t, x, y) w_{l}(y) d y d S=\int_{\partial \omega} v_{k}(x) h_{l}(t, x) d S \\
& \text { in case } B=\partial_{v}+\gamma I .
\end{aligned}
$$

Consequently,

$$
\left\langle\left(\mu_{k}+\Delta_{d}-q\right) u, \mathrm{v}_{k, l}\right\rangle_{L_{2}(\Omega)}=(-1)^{\vartheta} \int_{\partial \omega} \partial_{v}^{\vartheta} v_{k}(x) h_{l}(t, x) d S .
$$

By means of similar computations, we deduce

$$
\left\langle\left(\varrho_{l}+\partial_{y}^{2}\right) u, \mathbf{v}_{k, l}\right\rangle_{L_{2}(\Omega)}=(-1)^{\vartheta_{0}+1} w_{l}^{\left(\vartheta_{0}\right)}(0) g_{0 ; k}(t)+(-1)^{\vartheta_{1}} w_{l}^{\left(\vartheta_{1}\right)}(1) g_{1 ; k}(t) .
$$

Plugging (19) and (20) into (18), we obtain (15).

Remark 1. In subsequent proofs of uniqueness of solutions of IP1 and IP2, we need the formula for Fourier coefficients of the solution of (1)-(4) only in the case $F=0, \varphi_{j}=0, j \in\{0 ;\lfloor\alpha\rfloor\}$. However, a corresponding formula in case of nonvanishing $F$ and $\varphi_{j}$ can be derived in a similar manner, with some additions.

\subsection{Two Basic Lemmas}

The analysis of IP1 and IP2 essentially uses two lemmas that we state below.

Lemma 3. Let $\alpha \in(0,1) \cup(1,2), m \in \mathbb{N}, \delta \in(0, T)$ and a family of functions $G_{K} \in L_{1}(0, T-\delta)$, $K \in \mathbb{N}^{m}$, be bounded in $L_{1}(0, T-\delta)$. Moreover, let a function $\Lambda: \mathbb{N}^{m} \mapsto \mathbb{R}$ be such that $\Lambda(K) \rightarrow \infty$ as $|K| \rightarrow \infty$, where $|K|=\sqrt{K_{1}^{2}+\ldots+K_{m}^{2}}, K=\left(K_{1}, \ldots, K_{m}\right)$. Then, for any $N \in \mathbb{N}$,

$$
\begin{aligned}
& \int_{0}^{T-\delta} e_{\alpha}(-\Lambda(K) ; T-\tau) G_{K}(\tau) d \tau=\sum_{n=1}^{N} \frac{(-1)^{n}}{\Gamma(-n \alpha)} \int_{0}^{T-\delta} \frac{G_{K}(\tau) d \tau}{(T-\tau)^{n \alpha+1}} \frac{1}{\Lambda(K)^{n+1}} \\
& +O\left(\frac{1}{\Lambda(K)^{N+2}}\right) \text { as }|K| \rightarrow \infty
\end{aligned}
$$

Proof. The proof is based on the following expansion of the function $E_{\alpha, \alpha}$ :

$$
\begin{aligned}
& E_{\alpha, \alpha}(-z)=\sum_{n=1}^{N} \frac{(-1)^{n}}{\Gamma(-n \alpha) z^{n+1}}+\hat{R}_{N}(z), \quad z>0, N \in \mathbb{N}, \\
& \exists \hat{z}_{N}, \hat{C}_{N}>0:\left|\hat{R}_{N}(z)\right| \leq \frac{\hat{C}_{N}}{z^{N+2}} \text { for } z>\hat{z}_{N}
\end{aligned}
$$

(see Reference [27], Formula (1.8.28)). By the definition of $e_{\alpha}$, we have $e_{\alpha}(-\lambda(K) ; T-\tau)=$ $(T-\tau)^{\alpha-1} E_{\alpha, \alpha}\left(-\Lambda(K)(T-\tau)^{\alpha}\right)$. Therefore, by means of (22), we deduce

$$
\int_{0}^{T-\delta} e_{\alpha}(-\Lambda(K) ; T-\tau) G_{K}(\tau) d \tau=\sum_{n=1}^{N} \frac{(-1)^{n}}{\Gamma(-n \alpha)} \int_{0}^{T-\delta} \frac{G_{K}(\tau) d \tau}{(T-\tau)^{n \alpha+1}} \frac{1}{\Lambda(K)^{n+1}}+R_{N}(K)
$$


in case $\Lambda(K)>0$, where

$$
R_{N}(K)=\int_{0}^{T-\delta}(T-\tau)^{\alpha-1} \hat{R}_{N}\left(\Lambda(K)(T-\tau)^{\alpha}\right) G_{K}(\tau) d \tau
$$

Due to (23), it holds that

$$
\left|R_{N}(K)\right| \leq \frac{\hat{C}_{N}}{\lambda(K)^{N+2}} \int_{0}^{T-\delta} \frac{\left|G_{K}(\tau)\right| d \tau}{(T-\tau)^{(N+1) \alpha+1}} \leq \frac{\hat{C}_{N}}{\Lambda(K)^{N+2}} \frac{\sup _{s \in \mathbb{N}^{m}}\left\|G_{s}\right\|_{L_{1}(0, T-\delta)}}{\delta^{(N+1) \alpha+1}},
$$

provided that $|K|$ is sufficiently large to guarantee that $\Lambda(K)>\hat{z}_{N} \delta^{-\alpha}$. This proves (21).

Lemma 4. Let $\alpha \in(0,1) \cup(1,2), g \in L_{1}(0, T-\delta)$ for some $\delta \in(0, T)$ and

$$
\frac{1}{\Gamma(-n \alpha)} \int_{0}^{T-\delta} \frac{g(\tau) d \tau}{(T-\tau)^{n \alpha+1}}=0, n \in \mathbb{N} .
$$

Then, $g=0$.

This lemma, in a bit more general case, was proved in paper [22], pp. 1688-1690. A sketch of the proof is as follows. There exist $n_{0}, n_{1} \in \mathbb{N}$ such that $\frac{1}{\Gamma(-n \alpha)} \neq 0$ for $n=n_{0}+n_{1} n^{\prime}, n^{\prime} \in\{0\} \cup \mathbb{N}$. The set of equalities $\frac{1}{\Gamma(-n \alpha)} \int_{0}^{T-\delta} \frac{g(\tau) d \tau}{(T-\tau)^{n \alpha+1}}=0$ for $n=n_{0}+$ $n_{1} n^{\prime}$ is transformed by the substitution $s=(T-\tau)^{-n_{1} \alpha}$ to $\int_{a}^{b} s^{n^{\prime}} \widehat{g}(s) d s=0, n^{\prime} \in\{0\} \cup \mathbb{N}$, where $a$ and $b$ are some numbers, and $\widehat{g}$ is expressed in terms of $g$ by an explicit formula. This implies $\int_{a}^{b} P(s) \widehat{g}(s) d s=0$ for any polynomial $P$ and, due to the Weierstrass theorem, $\int_{a}^{b} Z(s) \widehat{g}(s) d s=0$ for any $Z \in C[a, b]$. This yields $\widehat{g}=0$ and, in turn, $g=0$.

\section{Uniqueness for IP1}

We start this section with a technical lemma.

Lemma 5. The following relations are valid:

$$
\begin{aligned}
& w_{l}^{\prime}(0)=l \pi, w_{l}^{\prime}(1)=(-1)^{l} l \pi \quad \text { in case } B_{0}=I, B_{1}=I, \\
& w_{l}^{\prime}(0)=p_{l}, w_{l}(1)=\frac{(-1)^{l+1} p_{l}}{\sqrt{\gamma_{1}^{2}+p_{l}^{2}}} \text { in case } B_{0}=I, B_{1}=\partial_{y}+\gamma_{1} I, \\
& w_{l}(0)=1, w_{l}(1)=(-1)^{l+1} \sqrt{\frac{\gamma_{0}^{2}+p_{l}^{2}}{\gamma_{1}^{2}+p_{l}^{2}}} \quad \text { in case } B_{0}=\partial_{y}-\gamma_{0} I, B_{1}=\partial_{y}+\gamma_{1} I,
\end{aligned}
$$

where the numbers $p_{l}$ are defined in (9) and (10). In the special case $B_{0}=\partial_{y}-\gamma_{0} I, B_{1}=\partial_{y}+\gamma_{1} I$, $\gamma_{0}=\gamma_{1}=0$, it holds that $p_{1}=0$, and we equate the undetermined quotient $\frac{\gamma_{0}^{2}+p_{1}^{2}}{\gamma_{1}^{2}+p_{1}^{2}}$ with 1 .

Proof. Formulas (25) immediately follow from (8). In case $B_{0}=I, B_{1}=\partial_{y}+\gamma_{1} I$, from (9), we get $w_{l}^{\prime}(0)=p_{l}, w_{l}(1)=\sin p_{l}$ and $\cot p_{l}=-\frac{\gamma_{1}}{p_{l}}$. Since $p_{l} \in((l-1) \pi, l \pi)$, we obtain $w_{l}(1)=(-1)^{l+1} \sqrt{\frac{1}{\cot ^{2} p_{l}+1}}=\frac{(-1)^{l+1} p_{l}}{\sqrt{\gamma_{1}^{2}+p_{l}^{2}}}$. Finally, we consider the case $B_{0}=\partial_{y}-\gamma_{0} I$, $B_{1}=\partial_{y}+\gamma_{1} I$. If $\gamma_{0}=\gamma_{1}=0$, then the Formulas (27) easily follow from (10). If $\gamma_{0}+\gamma_{1}>0$, then, from (10), we get $w_{l}(0)=1, w_{l}(1)=\cot p_{l} \sin p_{l}+\gamma_{0} \frac{\sin p_{l}}{p_{l}}$ and $\cot p_{l}=\frac{p_{l}^{2}-\gamma_{0} \gamma_{1}}{\left(\gamma_{0}+\gamma_{1}\right) p_{l}}$. 
Substituting $\sin p_{l}$ by $(-1)^{l+1} \sqrt{\frac{1}{\cot ^{2} p_{l}+1}}$ and $\cot p_{l}$ by $\frac{p_{l}^{2}-\gamma_{0} \gamma_{1}}{\left(\gamma_{0}+\gamma_{1}\right) p_{l}}$ in the formula of $w_{l}(1)$, after some computations, we arrive at $w_{l}(1)=(-1)^{l+1} \sqrt{\frac{\gamma_{0}^{2}+p_{l}^{2}}{\gamma_{1}^{2}+p_{l}^{2}}}$.

Now, we formulate and prove the uniqueness statement for IP1.

Theorem 1. Let $\alpha \in(0,1) \cup(1,2)$ and $u \in \mathcal{U}_{\alpha}$ satisfy (1)-(3) with $F=0, \varphi_{j}=0, j \in\{0 ;\lfloor\alpha\rfloor\}$, $h=0$. Moreover, assume that

$$
\left.B_{0} u(t, x, y)\right|_{y=0}=0,\left.\quad B_{1} u(t, x, y)\right|_{y=1}=0, \quad t \in(T-\delta, T), x \in \omega,
$$

for some $\delta \in(0, T)$ and

$$
u(T, x, y)=0, \quad(x, y) \in \Omega
$$

Then, $u=0$.

Proof. Let us define the functions $g_{0}:=\left.B_{0} u\right|_{(0, T) \times \omega \times\{0\}}, g_{1}:=\left.B_{1} u\right|_{(0, T) \times \omega \times\{1\}}$. Then, Proposition 1 implies that

$$
\begin{aligned}
& u_{k, l}(t)=e_{\alpha}\left(-\lambda_{k, l} ; t\right) *\left((-1)^{\vartheta_{0}+1} w_{l}^{\left(\vartheta_{0}\right)}(0) g_{0 ; k}(t)+(-1)^{\vartheta_{1}} w_{l}^{\left(\vartheta_{1}\right)}(1) g_{1 ; k}(t)\right), \\
& t \in[0, T], k \in \mathcal{K}, l \in \mathbb{N}
\end{aligned}
$$

where $\vartheta_{0}, \vartheta_{1}$ are given by (16). It suffices to show that $g_{0 ; k}=0, g_{1 ; k}=0, k \in \mathcal{K}$, because then, from (28), we have $u_{k, l}=0, k \in \mathcal{K}, l \in \mathbb{N}$ and, due to the completeness of the system $\mathrm{v}_{k, l}, k \in \mathcal{K}, l \in \mathbb{N}$, in $L_{2}(\Omega)$, we obtain $u=0$.

So, let us choose $k \in \mathcal{K}$ and prove that $g_{0 ; k}=0, g_{1 ; k}=0$. Since $\left.g_{0}\right|_{(T-\delta, T) \times \omega}=$ $\left.g_{1}\right|_{(T-\delta, T) \times \omega}=0$, we have $\left.g_{0 ; k}\right|_{(T-\delta, T)}=\left.g_{1 ; k}\right|_{(T-\delta, T)}=0$. Moreover, by $u(T, \cdot)=0$, it holds that $u_{k, l}(T)=0$. From (28), we obtain the following relations:

$$
\begin{aligned}
& 0=\int_{0}^{T-\delta} e_{\alpha}\left(-\lambda_{k, l} ; T-\tau\right) \\
& \times\left((-1)^{\vartheta_{0}+1} w_{l}^{\left(\vartheta_{0}\right)}(0) g_{0 ; k}(\tau)+(-1)^{\vartheta_{1}} w_{l}^{\left(\vartheta_{1}\right)}(1) g_{1 ; k}(\tau)\right) d \tau, \quad l \in \mathbb{N} .
\end{aligned}
$$

Firstly, let us consider the simplest case $B_{0}=I, B_{1}=I$. Then, $\left(\vartheta_{0}, \vartheta_{1}\right)=(1,1)$ and by (25), the relation (29) transforms to

$$
\int_{0}^{T-\delta} e_{\alpha}\left(-\lambda_{k, l} ; T-\tau\right)\left(g_{0 ; k}(\tau)+(-1)^{l+1} g_{1 ; k}(\tau)\right) d \tau=0, \quad l \in \mathbb{N} .
$$

Therefore,

$$
\begin{aligned}
& \int_{0}^{T-\delta} e_{\alpha}\left(-\lambda_{k, 2 i} ; T-\tau\right)\left(g_{0 ; k}(\tau)-g_{1 ; k}(\tau)\right) d \tau=0, \quad i \in \mathbb{N}, \\
& \int_{0}^{T-\delta} e_{\alpha}\left(-\lambda_{k, 2 i-1} ; T-\tau\right)\left(g_{0 ; k}(\tau)+g_{1 ; k}(\tau)\right) d \tau=0, \quad i \in \mathbb{N} .
\end{aligned}
$$

Since $\lambda_{k, 2 i}=\mu_{k}+\varrho_{2 i} \rightarrow \infty$ as $i \rightarrow \infty$ and $g_{0 ; k}-g_{1 ; k}$ is independent of $i$, we can apply Lemma 3 with the parameters $K=i$ and $\Lambda(K)=\lambda_{k, 2 i}$ to the integral in (30). Let us choose $N \in \mathbb{N}$. We have

$$
\sum_{n=1}^{N} \frac{(-1)^{n}}{\Gamma(-n \alpha)} \int_{0}^{T-\delta} \frac{\left(g_{0 ; k}(\tau)-g_{1 ; k}(\tau)\right) d \tau}{(T-\tau)^{n \alpha+1}} \frac{1}{\lambda_{k, 2 i}^{n+1}}=O\left(\frac{1}{\lambda_{k, 2 i}^{N+2}}\right) \text { as } i \rightarrow \infty .
$$


Since coefficients of the terms $\frac{1}{\lambda_{k, 2 i}^{n+1}}$ in the left-hand side of (32) are constant in $i$, (32) can only be valid if these coefficients vanish. Consequently,

$$
\frac{1}{\Gamma(-n \alpha)} \int_{0}^{T-\delta} \frac{\left(g_{0 ; k}(\tau)-g_{1 ; k}(\tau)\right) d \tau}{(T-\tau)^{n \alpha+1}}=0
$$

for any $n=1, \ldots, N$. Since $N \in \mathbb{N}$ was chosen arbitrarily, (33) holds for any $n \in \mathbb{N}$. Lemma 4 implies $g_{0 ; k}-g_{1 ; k}=0$. Similarly, from (31), we deduce $g_{0 ; k}+g_{1 ; k}=0$. Consequently, $g_{0 ; k}=0$ and $g_{1 ; k}=0$.

Secondly, we consider the case $B_{0}=\partial_{y}-\gamma_{0} I, B_{1}=\partial_{y}+\gamma_{1} I$. Then, $\left(\vartheta_{0}, \vartheta_{1}\right)=(0,0)$, and, in view of (27) from (29), we get the relations

$$
\int_{0}^{T-\delta} e_{\alpha}\left(-\lambda_{k, l} ; T-\tau\right)\left(-g_{0 ; k}(\tau)+(-1)^{l+1} z_{l} g_{1 ; k}(\tau)\right) d \tau=0, \quad l \in \mathbb{N},
$$

where $z_{l}=\sqrt{\frac{\gamma_{0}^{2}+p_{l}^{2}}{\gamma_{1}^{2}+p_{l}^{2}}}$. The sequence $z_{l}, l \in \mathbb{N}$, is bounded. Therefore, the family of functions $g_{0 ; k}+(-1)^{l} z_{l} g_{1 ; k}, l \in \mathbb{N}$, is bounded in $L_{1}(0, T-\delta)$. Let $N \in \mathbb{N}$. Applying Lemma 3 with $K=l, \Lambda(K)=\lambda_{k, l}$, we obtain

$$
\sum_{n=1}^{N} \frac{(-1)^{n}}{\Gamma(-n \alpha)} \int_{0}^{T-\delta} \frac{\left(g_{0 ; k}(\tau)+(-1)^{l} z_{l} g_{1 ; k}(\tau)\right) d \tau}{(T-\tau)^{n \alpha+1}} \frac{1}{\lambda_{k, l}^{n+1}}=O\left(\frac{1}{\lambda_{k, l}^{N+2}}\right) \text { as } l \rightarrow \infty .
$$

Let us prove by means of the mathematical induction that the following equalities

$$
\frac{1}{\Gamma(-n \alpha)} \int_{0}^{T-\delta} \frac{g_{0 ; k}(\tau) d \tau}{(T-\tau)^{n \alpha+1}}=0, \frac{1}{\Gamma(-n \alpha)} \int_{0}^{T-\delta} \frac{g_{1 ; k}(\tau) d \tau}{(T-\tau)^{n \alpha+1}}=0
$$

are valid for $n=1, \ldots, N$. To this end, we suppose that either $\hat{n}=1$ or $\hat{n} \in\{2 ; \ldots ; N\}$ and (35) are valid for $n=1, \ldots, \hat{n}-1$. The aim is to show that, then, (35) holds for $n=\hat{n}$, too.

According to the supposition, (34) transforms to

$$
\sum_{n=\hat{n}}^{N} \frac{(-1)^{n}}{\Gamma(-n \alpha)} \int_{0}^{T-\delta} \frac{\left(g_{0 ; k}(\tau)+(-1)^{l} z_{l} g_{1 ; k}(\tau)\right) d \tau}{(T-\tau)^{n \alpha+1}} \frac{1}{\lambda_{k, l}^{n+1}}=O\left(\frac{1}{\lambda_{k, l}^{N+2}}\right) \text { as } l \rightarrow \infty .
$$

Multiplying (36) by $\lambda_{k, l}^{\hat{n}+1}$ and extracting the term $n=\hat{n}$, we deduce

$$
\frac{1}{\Gamma(-\hat{n} \alpha)} \int_{0}^{T-\delta} \frac{\left(g_{0 ; k}(\tau)+(-1)^{l} z_{l} g_{1 ; k}(\tau)\right) d \tau}{(T-\tau)^{\hat{n} \alpha+1}}=O\left(\frac{1}{\lambda_{k, l}}\right) \text { as } l \rightarrow \infty .
$$

Now, we distinguish between the cases of even and odd $l$. We have

$$
\begin{aligned}
& \frac{1}{\Gamma(-\hat{n} \alpha)} \int_{0}^{T-\delta} \frac{\left(g_{0 ; k}(\tau)+z_{2 i g_{1 ; k}}(\tau)\right) d \tau}{(T-\tau)^{\hat{n} \alpha+1}}=O\left(\frac{1}{\lambda_{k, 2 i}}\right) \text { as } i \rightarrow \infty, \\
& \frac{1}{\Gamma(-\hat{n} \alpha)} \int_{0}^{T-\delta} \frac{\left(g_{0 ; k}(\tau)-z_{2 i-1} g_{1 ; k}(\tau)\right) d \tau}{(T-\tau)^{\hat{n} \alpha+1}}=O\left(\frac{1}{\lambda_{k, 2 i-1}}\right) \text { as } i \rightarrow \infty .
\end{aligned}
$$


Since $p_{l} \rightarrow \infty$ as $l \rightarrow \infty$, the number $z_{l}=\sqrt{\frac{\gamma_{0}^{2}+p_{l}^{2}}{\gamma_{1}^{2}+p_{l}^{2}}}$ approaches 1 as $l \rightarrow \infty$. Passing to the limit $i \rightarrow \infty$ in (37) and (38), we obtain

$$
\frac{1}{\Gamma(-\hat{n} \alpha)} \int_{0}^{T-\delta} \frac{\left(g_{0 ; k}(\tau) \pm g_{1 ; k}(\tau)\right) d \tau}{(T-\tau)^{\hat{n} \alpha+1}}=0 .
$$

This implies (35) in case $n=\hat{n}$. The induction is complete.

We have shown that (35) are valid for $n=1, \ldots, N$. Since $N \in \mathbb{N}$ is arbitrary, (35) holds for any $n \in \mathbb{N}$. Lemma 4 implies $g_{0 ; k}=0$ and $g_{1 ; k}=0$.

Finally, we deal with the case $B_{0}=I, B_{1}=\partial_{y}+\gamma_{1} I$. Then, $\left(\vartheta_{0}, \vartheta_{1}\right)=(1,0)$, and, by means of (26) from (29), we obtain

$$
\begin{aligned}
& p_{l} \int_{0}^{T-\delta} e_{\alpha}\left(-\lambda_{k, l} ; T-\tau\right) g_{0 ; k}(\tau) d \tau \\
& +(-1)^{l+1} \xi_{l} \int_{0}^{T-\delta} e_{\alpha}\left(-\lambda_{k, l} ; T-\tau\right) g_{1 ; k}(\tau) d \tau=0, \quad l \in \mathbb{N},
\end{aligned}
$$

where $\xi_{l}=\frac{p_{l}}{\sqrt{\gamma_{1}^{2}+p_{l}^{2}}}$. The addends at the left-hand side of (39) behave differently in the process $l \rightarrow \infty$ because $p_{l} \rightarrow \infty$ but $\xi_{l}$ is bounded. Let us take $N \in \mathbb{N}$ and apply Lemma 3 separately to both integrals in (39). Since $\lambda_{k, l}=\mu_{k}+p_{l}^{2}$, we obtain

$$
\begin{aligned}
& \sum_{n=1}^{N}\left[\frac{(-1)^{n}}{\Gamma(-n \alpha)} \int_{0}^{T-\delta} \frac{g_{0 ; k}(\tau) d \tau}{(T-\tau)^{n \alpha+1}} \frac{p_{l}}{\left(\mu_{k}+p_{l}^{2}\right)^{n+1}}\right. \\
& \left.+\frac{(-1)^{n}}{\Gamma(-n \alpha)} \int_{0}^{T-\delta} \frac{g_{1 ; k}(\tau) d \tau}{(T-\tau)^{n \alpha+1}} \frac{(-1)^{l+1} \xi_{l}}{\left(\mu_{k}+p_{l}^{2}\right)^{n+1}}\right]=O\left(\frac{p_{l}}{\left(\mu_{k}+p_{l}^{2}\right)^{N+2}}\right) \text { as } l \rightarrow \infty
\end{aligned}
$$

Again, we use the induction to prove that the equalities (35) are valid for $n=1, \ldots, N$. We suppose that either $\hat{n}=1$ or $\hat{n} \in\{2 ; \ldots ; N\}$ and (35) are valid for $n=1, \ldots, \hat{n}-1$. We have to show that, then, (35) holds for $n=\hat{n}$, too.

By the supposition, we have

$$
\begin{aligned}
& \sum_{n=\hat{n}}^{N}[\underbrace{\frac{(-1)^{n}}{\Gamma(-n \alpha)} \int_{0}^{T-\delta} \frac{g_{0 ; k}(\tau) d \tau}{(T-\tau)^{n \alpha+1}}}_{s_{n, 1}} \frac{p_{l}}{\left(\mu_{k}+p_{l}^{2}\right)^{n+1}} \\
& +\underbrace{\frac{(-1)^{n}}{\Gamma(-n \alpha)} \int_{0}^{T-\delta} \frac{g_{1 ; k}(\tau) d \tau}{(T-\tau)^{n \alpha+1}}}_{s_{n, 2}} \frac{(-1)^{l+1} \xi_{l}}{\left(\mu_{k}+p_{l}^{2}\right)^{n+1}}]=O\left(\frac{p_{l}}{\left(\mu_{k}+p_{l}^{2}\right)^{N+2}}\right) \text { as } l \rightarrow \infty .
\end{aligned}
$$

Multiplying (40) by $\frac{\left(\mu_{k}+p_{l}^{2}\right)^{\hat{n}+1}}{p_{l}}$ and expressing $s_{\hat{n}, 1}$, we obtain $s_{\hat{n}, 1}=O\left(\frac{1}{p_{l}}\right)$ as $l \rightarrow \infty$. This yields $s_{\hat{n}, 1}=0$ and

$$
\frac{1}{\Gamma(-\hat{n} \alpha)} \int_{0}^{T-\delta} \frac{g_{0 ; k}(\tau) d \tau}{(T-\tau)^{\hat{n} \alpha+1}}=0
$$

Then, multiplying (40) by $\frac{\left(\mu_{k}+p_{l}^{2}\right)^{n+1}}{(-1)^{l+1} \xi_{l}}$ and taking the relation $\xi_{l} \rightarrow 1$ as $l \rightarrow \infty$ into account, we deduce $s_{\hat{n}, 2}=O\left(\frac{1}{p_{l}}\right)$ as $l \rightarrow \infty$. Therefore, $s_{\hat{n}, 2}=0$, and

$$
\frac{1}{\Gamma(-\hat{n} \alpha)} \int_{0}^{T-\delta} \frac{g_{1 ; k}(\tau) d \tau}{(T-\tau)^{\hat{n} \alpha+1}}=0
$$


We have shown that (35) is valid in case $n=\hat{n}$. The induction is complete. We finish the proof as in the previous case.

As a corollary of Theorem 1, we deduce the uniqueness of a solution of an inverse problem in an interval. This problem consists in finding a function $u(t, y)$ that satisfies the equation $D_{t}^{\alpha-\lfloor\alpha\rfloor}\left[\partial_{t}^{\lfloor\alpha\rfloor} u-\varphi_{\lfloor\alpha\rfloor}\right](t, y)-\left(\partial_{y}^{2}-q\right) u(t, y)=F(t, y)$ for $t \in(0, T), y \in(0,1)$, the initial condition(s) $\left.\partial_{t}^{j} u(t, y)\right|_{t=0}=\varphi_{j}(y)$ for $y \in(0,1), j \in\{0 ;\lfloor\alpha\rfloor\}$, the partly given boundary conditions $\left.B_{0} u(t, y)\right|_{y=0}=g_{0}(t),\left.B_{1} u(t, y)\right|_{y=1}=g_{1}(t)$ for $t \in(T-\delta, T)$, and the final condition $u(T, y)=\psi(y)$ for $y \in(0,1)$.

Corollary 1. Let $\alpha \in(0,1) \cup(1,2), u \in C^{\lfloor\alpha\rfloor}\left([0, T] ; L_{2}(0,1)\right)$ and $\exists p \in(1, \infty): u \in$ $L_{p}\left((0, T) ; H^{2}(0,1)\right), \partial_{t}^{\lfloor\alpha\rfloor} u \in{ }_{0} H_{p}^{\alpha-\lfloor\alpha\rfloor}\left((0, T) ; L_{2}(0,1)\right)$. Assume that

$$
\begin{aligned}
& D_{t}^{\alpha-\lfloor\alpha\rfloor} \partial_{t}^{\lfloor\alpha\rfloor} u(t, y)-\left(\partial_{y}^{2}-q\right) u(t, y)=0, \quad t \in(0, T), \quad y \in(0,1), \\
& \left.\partial_{t}^{j} u(t, y)\right|_{t=0}=0, \quad y \in(0,1), \quad j \in\{0 ;\lfloor\alpha\rfloor\}, \\
& \left.B_{0} u(t, y)\right|_{y=0}=0,\left.\quad B_{1} u(t, y)\right|_{y=1}=0, \quad t \in(T-\delta, T)
\end{aligned}
$$

for some $\delta \in(0, T)$ and

$$
u(T, y)=0, \quad y \in(0,1)
$$

Then, $u=0$.

Proof. Let us define $\widetilde{u}(t, x, y)=u(t, y) \sin (\pi x), t \in[0, T],(x, y) \in \bar{\Omega}$, where $\Omega=\omega \times$ $(0,1), \omega=(0,1)$. Then, $\widetilde{u} \in \mathcal{U}_{\alpha}$,

$$
\begin{aligned}
& D_{t}^{\alpha-\lfloor\alpha\rfloor} \partial_{t}^{\lfloor\alpha\rfloor} \widetilde{u}(t, x, y)-(\Delta-\widetilde{q}) \widetilde{u}(t, x, y)=0, t \in(0, T), \quad(x, y) \in \Omega, \\
& \left.\partial_{t}^{j} \widetilde{u}(t, x, y)\right|_{t=0}=0, \quad(x, y) \in \Omega, \quad j \in\{0 ;\lfloor\alpha\rfloor\}, \\
& \widetilde{u}(t, x, y)=0, \quad t \in(0, T), x \in \partial \omega, y \in(0,1), \\
& \left.B_{0} \widetilde{u}(t, x, y)\right|_{y=0}=0,\left.\quad B_{1} \widetilde{u}(t, x, y)\right|_{y=1}=0, \quad t \in(T-\delta, T), x \in \omega, \\
& \widetilde{u}(T, x, y)=0, \quad(x, y) \in \Omega,
\end{aligned}
$$

where $\widetilde{q}=q-\pi^{2}$. Theorem 1 implies $\widetilde{u}=0$. Hence, $u=0$.

\section{Uniqueness for IP2}

In this section, we consider IP2 in two cases: $\Omega$ is a $d+1$-dimensional cube, and $\Omega$ is a circular cylinder. For the sake of simplicity, we will be limited to problems with Dirichlet boundary conditions.

\section{1. $d+1-C u b e$}

We are going to prove the uniqueness for IP2 when $\Omega$ is the $d+1$-cube, i.e., $\Omega=\omega \times(0,1)$, $\omega=(0,1)^{d}$. The case of hyperrectangle $\Omega=\left(0, a_{1}\right) \times \ldots \times\left(0, a_{d}\right) \times(0,1), a_{j}>0$, can be handled in a similar manner, with little modifications.

In case $\omega=(0,1)^{d}$ and $B=I$, the solution of the eigenvalue problem (6) reads

$$
\begin{aligned}
& \mu_{k}=\left(k_{1} \pi\right)^{2}+\ldots+\left(k_{d} \pi\right)^{2}+q, \quad v_{k}(x)=\prod_{j=1}^{d} \sin \left(k_{j} \pi x_{j}\right), \\
& k=\left(k_{1}, \ldots, k_{d}\right) \in \mathcal{K}=\mathbb{N}^{d} .
\end{aligned}
$$


Theorem 2. Let $\alpha \in(0,1) \cup(1,2)$ and $\omega=(0,1)^{d}$. Then, the following statement is valid. If a function $u \in \mathcal{U}_{\alpha}$ satisfies (1) and (2) with $F=0, \varphi_{j}=0, j \in\{0 ;\lfloor\alpha\rfloor\}$, fulfills the conditions

$$
\begin{aligned}
& u(t, x, y)=0, \quad t \in(T-\delta, T), x \in \partial \omega, y \in(0,1), \\
& u(t, x, 0)=0, \quad u(t, x, 1)=0, \quad t \in(T-\delta, T), x \in \omega,
\end{aligned}
$$

for some $\delta \in(0, T)$ and

$$
u(T, x, y)=0, \quad(x, y) \in \Omega,
$$

then $u=0$.

Proof. By Proposition 1 and (25), we have

$$
\begin{aligned}
& \left\langle u(t, \cdot, \cdot), \mathrm{v}_{k, l}\right\rangle_{L_{2}(\Omega)}=e_{\alpha}\left(-\lambda_{k, l} ; t\right) *\left(-\int_{\partial \omega} \partial_{v} v_{k}(x)\left\langle u(t, x, \cdot), w_{l}\right\rangle_{L_{2}(0,1)} d S\right. \\
& \left.+\pi l\left\langle u(t, \cdot, 0), v_{k}\right\rangle_{L_{2}(\omega)}-(-1)^{l} \pi l\left\langle u(t, \cdot, 1), v_{k}\right\rangle_{L_{2}(\omega)}\right), \\
& t \in[0, T], \quad k \in \mathbb{N}^{d}, l \in \mathbb{N} .
\end{aligned}
$$

Since $\Omega$ is the cube, it is reasonable to unify the notation in $x$ and $y$ directions. Therefore, we denote

$$
\begin{aligned}
& x_{d+1}=y, \quad k_{d+1}=l, \\
& X=\left(x_{1}, \ldots, x_{d+1}\right), K=\left(k_{1}, \ldots, k_{d+1}\right),|K|=\sqrt{k_{1}^{2}+\ldots+k_{d+1}^{2}}, \\
& V_{K}(X)=\prod_{j=1}^{d+1} \sin \left(k_{j} \pi x_{j}\right) .
\end{aligned}
$$

The relation (42) transforms to

$$
\begin{aligned}
& \left\langle u(t, \cdot, \cdot), V_{K}\right\rangle_{L_{2}(\Omega)}=e_{\alpha}\left(-\pi^{2}|K|^{2}-q ; t\right) \\
& *\left(-\int_{0}^{1} \sin \left(k_{d+1} \pi x_{d+1}\right) \int_{\partial \omega} \partial_{v}\left[\prod_{s=1}^{d} \sin \left(k_{s} \pi x_{s}\right)\right] u\left(t, x, x_{d+1}\right) d S d x_{d+1}\right. \\
& \left.+k_{d+1} \pi\left[\int_{\Omega} \prod_{j=1}^{d} \sin \left(k_{j} \pi x_{j}\right) u(t, x, 0) d x-(-1)^{l} \int_{\Omega} \prod_{j=1}^{d} \sin \left(k_{j} \pi x_{j}\right) u(t, x, 1) d x\right]\right), \\
& t \in[0, T], \quad K \in \mathbb{N}^{d+1} .
\end{aligned}
$$

Let us introduce the following notation for the traces of $u$ on the faces of $\Omega$ :

$$
\begin{aligned}
& h_{0, j}\left(t, x_{1}, \ldots, x_{j-1}, x_{j+1}, \ldots, x_{d+1}\right)=u\left(t, x_{1}, \ldots, x_{j-1}, 0, x_{j+1}, \ldots, x_{d+1}\right), \\
& h_{1, j}\left(t, x_{1}, \ldots, x_{j-1}, x_{j+1}, \ldots, x_{d+1}\right)=u\left(t, x_{1}, \ldots, x_{j-1}, 1, x_{j+1}, \ldots, x_{d+1}\right), \\
& j=1, \ldots, d+1
\end{aligned}
$$


and define the $t$-dependent functions

$$
\begin{aligned}
& h_{0, j ; k_{1}, \ldots, k_{j-1}, k_{j+1}, \ldots, k_{d+1}}(t)=\int_{(0,1)^{d}} h_{0, j}\left(t, x_{1}, \ldots, x_{j-1}, x_{j+1}, \ldots, x_{d+1}\right) \\
& \times \prod_{\substack{s=1 \\
s \neq j}}^{d+1} \sin \left(k_{s} \pi x_{s}\right) d x_{1} \ldots d x_{j-1} d x_{j+1} \ldots d x_{d+1}, \\
& h_{1, j ; k_{1}, \ldots, k_{j-1}, k_{j+1}, \ldots, k_{d+1}}(t)=\int_{(0,1)^{d}} h_{1, j}\left(t, x_{1}, \ldots, x_{j-1}, x_{j+1}, \ldots, x_{d+1}\right) \\
& \times \prod_{\substack{s=1 \\
s \neq j}}^{d+1} \sin \left(k_{s} \pi x_{s}\right) d x_{1} \ldots d x_{j-1} d x_{j+1} \ldots d x_{d+1}, \\
& k_{1}, \ldots, k_{j-1}, k_{j+1}, \ldots k_{d+1} \in \mathbb{N}, \quad j=1, \ldots, d+1 .
\end{aligned}
$$

Then,

$$
\begin{aligned}
& -\int_{0}^{1} \sin \left(k_{d+1} \pi x_{d+1}\right) \int_{\partial \omega} \partial_{v}\left[\prod_{s=1}^{d} \sin \left(k_{s} \pi x_{s}\right)\right] u\left(t, x, x_{d+1}\right) d S d x_{d+1} \\
& =\int_{0}^{1} \sin \left(k_{d+1} \pi x_{d+1}\right) \sum_{j=1}^{d} \int_{(0,1)^{d-1}} \prod_{\substack{s=1 \\
s \neq j}}^{d} \sin \left(k_{s} \pi x_{s}\right) k_{j} \pi \\
& \times\left(h_{0, j}\left(t, x_{1}, \ldots, x_{j-1}, x_{j+1}, \ldots, x_{d+1}\right)\right. \\
& \left.-(-1)^{k_{j}} h_{1, j}\left(t, x_{1}, \ldots, x_{j-1}, x_{j+1}, \ldots, x_{d+1}\right)\right) d x_{1} \ldots d x_{j-1} d x_{j+1} \ldots d x_{d+1} \\
& =\sum_{j=1}^{d} k_{j} \pi\left(h_{0, j ; k_{1}, \ldots, k_{j-1}, k_{j+1}, \ldots, k_{d+1}}(t)-(-1)^{k_{j}} h_{1, j ; k_{1}, \ldots, k_{j-1}, k_{j+1}, \ldots, k_{d+1}}(t)\right) .
\end{aligned}
$$

Plugging (46) into (43), we obtain

$$
\begin{aligned}
& \left\langle u(t, \cdot, \cdot), V_{K}\right\rangle_{L_{2}(\Omega)}=e_{\alpha}\left(-\pi^{2}|K|^{2}-q ; t\right) \\
& * \sum_{j=1}^{d+1} k_{j} \pi\left(h_{0, j ; k_{1}, \ldots, k_{j-1}, k_{j+1}, \ldots, k_{d+1}}(t)-(-1)^{k_{j}} h_{1, j ; k_{1}, \ldots, k_{j-1}, k_{j+1}, \ldots, k_{d+1}}(t)\right), \\
& t \in[0, T], \quad K \in \mathbb{N}^{d+1} .
\end{aligned}
$$

It suffices to prove that the family of functions (44) and (45) vanishes. Then, the assertion $u=0$ follows.

In view of the assumptions of the theorem, all functions of the family (44) and (45) are equal to zero for $t \in(T-\delta, T)$ and $\left\langle u(T, \cdot, \cdot), V_{K}\right\rangle_{L_{2}(\Omega)}=0$. Consequently, from (47), we have

$$
\begin{aligned}
& 0=\sum_{j=1}^{d+1} k_{j} \int_{0}^{T-\delta} e_{\alpha}\left(-\pi^{2}|K|^{2}-q ; T-\tau\right) \\
& \times\left(h_{0, j ; k_{1}, \ldots, k_{j-1}, k_{j+1}, \ldots, k_{d+1}}(\tau)-(-1)^{k_{j}} h_{1, j ; k_{1}, \ldots, k_{j-1}, k_{j+1}, \ldots, k_{d+1}}(\tau)\right) d \tau=0, \quad K \in \mathbb{N}^{d+1} .
\end{aligned}
$$


Let $N \in \mathbb{N}$. Since the family $h_{0, j ; k_{1}, \ldots, k_{j-1}, k_{j+1}, \ldots, k_{d+1}}-(-1)^{k_{j}} h_{1, j ; k_{1}, \ldots, k_{j-1}, k_{j+1}, \ldots, k_{d+1}}$ is bounded in $L_{1}(0, T-\delta)$, by means of Lemma 3 , we deduce

$$
\begin{aligned}
& \sum_{j=1}^{d+1} \sum_{n=1}^{N} a_{K ; n, j} \frac{k_{j}}{\left(\pi^{2}|K|^{2}+q\right)^{n+1}}=\sum_{j=1}^{d+1} k_{j} O\left(\frac{1}{\left(\pi^{2}|K|^{2}+q\right)^{N+2}}\right) \\
& =O\left(\frac{1}{\left(\pi^{2}|K|^{2}+q\right)^{N+3 / 2}}\right) \text { as }|K| \rightarrow \infty,
\end{aligned}
$$

where

$$
\begin{aligned}
& a_{K ; n, j}=\frac{(-1)^{n}}{\left(\pi^{2}\right)^{n+1}} \frac{1}{\Gamma(-n \alpha)} \\
& \times \int_{0}^{T-\delta} \frac{\left(h_{0, j ; k_{1}, \ldots, k_{j-1}, k_{j+1}, \ldots, k_{d+1}}(\tau)-(-1)^{k_{j}} h_{1, j ; k_{1}, \ldots, k_{j-1}, k_{j+1}, \ldots, k_{d+1}}(\tau)\right) d \tau}{(T-\tau)^{n \alpha+1}} .
\end{aligned}
$$

Next, we will prove by the induction that following equalities are valid:

$$
\left.\begin{array}{l}
\frac{1}{\Gamma(-n \alpha)} \int_{0}^{T-\delta} \frac{h_{0, j ; k_{1}, \ldots, k_{j-1}, k_{j+1}, \ldots, k_{d+1}}(\tau) d \tau}{(T-\tau)^{n \alpha+1}}=0, \\
\frac{1}{\Gamma(-n \alpha)} \int_{0}^{T-\delta} \frac{h_{1, j ; k_{1}, \ldots, k_{j-1}, k_{j+1}, \ldots, k_{d+1}}(\tau) d \tau}{(T-\tau)^{n \alpha+1}}=0 \\
\text { for any } j \in\{1, \ldots ; d+1\}, k_{1}, \ldots, k_{j-1}, k_{j+1}, \ldots, k_{d+1} \in \mathbb{N},
\end{array}\right\} \text {, }
$$

where $n$ runs from 1 to $N$.

Suppose that either $\hat{n}=1$ or $\hat{n} \in\{2 ; \ldots ; N\}$ and (49) are valid for $n=1, \ldots, \hat{n}-1$. We have to show that, then, (49) holds for $n=\hat{n}$, too.

The supposition yields $a_{K ; n, j}=0$ for $j \in\{1 ; \ldots ; d+1\}, K \in \mathbb{N}^{d+1}, n=1, \ldots, \hat{n}-1$. Hence, the relation (48) reduces to

$$
\sum_{n=\hat{n}}^{N} \sum_{j=1}^{d+1} a_{K ; n, j} \frac{k_{j}}{\left(\pi^{2}|K|^{2}+q\right)^{n+1}}=O\left(\frac{1}{\left(\pi^{2}|K|^{2}+q\right)^{N+3 / 2}}\right) \text { as }|K| \rightarrow \infty .
$$

Let us choose some $s \in\{1 ; \ldots ; d+1\}$, multiply (50) by $\frac{\left(\pi^{2}|K|^{2}+q\right)^{\hat{n}+1}}{k_{s}}$, and extract the addend $n=\hat{n}, j=s$. We obtain $a_{K ; \hat{n}, s}=O\left(\frac{1}{k_{s}}\right)$ as $k_{s} \rightarrow \infty$ for any fixed $k_{1}, \ldots, k_{s-1}, k_{s+1}, \ldots, k_{d+1} \in \mathbb{N}$. This implies

$$
\begin{aligned}
& \frac{1}{\Gamma(-\hat{n} \alpha)} \int_{0}^{T-\delta} \frac{\left(h_{0, s ; k_{1}, \ldots, k_{s-1}, k_{s+1}, \ldots, k_{d+1}}(\tau)-(-1)^{k_{s}} h_{1, s ; k_{1}, \ldots, k_{s-1}, k_{s+1}, \ldots, k_{d+1}}(\tau)\right) d \tau}{(T-\tau)^{\hat{n} \alpha+1}} \\
& =O\left(\frac{1}{k_{s}}\right) \text { as } k_{s} \rightarrow \infty
\end{aligned}
$$

for any fixed $k_{1}, \ldots, k_{s-1}, k_{s+1}, \ldots, k_{d+1} \in \mathbb{N}$. Setting in (51) $k_{s}=2 i$ and $k_{s}=2 i-1$ and letting $i \rightarrow \infty$, we reach the pair of relations

$$
\frac{1}{\Gamma(-\hat{n} \alpha)} \int_{0}^{T-\delta} \frac{\left(h_{0, s ; k_{1}, \ldots, k_{s-1}, k_{s+1}, \ldots, k_{d+1}}(\tau) \mp h_{1, s ; k_{1}, \ldots, k_{s-1}, k_{s+1}, \ldots, k_{d+1}}(\tau)\right) d \tau}{(T-\tau)^{\hat{n} \alpha+1}}=0
$$

for any $k_{1}, \ldots, k_{s-1}, k_{s+1}, \ldots, k_{d+1} \in \mathbb{N}$. Since $s \in\{1 ; \ldots ; d+1\}$ is arbitrary, (52) implies (49) for $n=\hat{n}$. The induction is complete.

We have proven that (49) are valid for $n=1, \ldots, N$. Since $N \in \mathbb{N}$ is arbitrary, (49) holds for all $n \in \mathbb{N}$. Lemma 4 implies that the functions (44) and (45) vanish. That is what we had to show. The theorem is proved. 


\subsection{Circular Cylinder}

In this subsection, we will study IP2 when $\Omega$ is the circular cylinder $\Omega=\omega \times(0,1)$, $\omega=\left\{x \in \mathbb{R}^{2}: x_{1}^{2}+x_{2}^{2}<1\right\}$.

So, let $\omega=\left\{x \in \mathbb{R}^{2}: x_{1}^{2}+x_{2}^{2}<1\right\}$ and $B=I$. We define $\mathcal{K}=\mathbb{N}^{2} \times\{1 ; 2\}$ and express the solution of the eigenvalue problem (6) as follows:

$$
\left.\begin{array}{l}
\mu_{k}=z_{k_{1}, k_{2}}^{2}+q, v_{k}(x)=J_{k_{1}}\left(z_{k_{1}, k_{2}} r\right) \cos \left(k_{1} \theta\right), k=\left(k_{1}, k_{2}, 1\right), k_{1}, k_{2} \in \mathbb{N}, \\
\mu_{k}=z_{k_{1}, k_{2}}^{2}+q, v_{k}(x)=J_{k_{1}}\left(z_{k_{1}, k_{2}} r\right) \sin \left(k_{1} \theta\right), k=\left(k_{1}, k_{2}, 2\right), k_{1}, k_{2} \in \mathbb{N}, \\
x=(r \cos \theta, r \sin \theta), 0 \leq r \leq 1,-\pi<\theta \leq \pi,
\end{array}\right\},
$$

where $J_{k_{1}}$ is the Bessel function of the first kind of the order $k_{1}$, and $z_{k_{1}, k_{2}}$ is the $k_{2}$-s positive root of $J_{k_{1}}$.

Lemma 6. The relations

$$
\begin{aligned}
& z_{k_{1}, k_{2}}=\pi\left(k_{2}+\frac{k_{1}}{2}-\frac{1}{4}\right)+O\left(\frac{1}{k_{2}}\right) \text { as } k_{2} \rightarrow \infty, \\
& \left.\frac{d}{d r} J_{k_{1}}\left(z_{k_{1}, k_{2}} r\right)\right|_{r=1}=\sqrt{\frac{2 z_{k_{1}, k_{2}}}{\pi}}\left[(-1)^{k_{2}}+O\left(\frac{1}{k_{2}}\right)\right] \text { as } k_{2} \rightarrow \infty
\end{aligned}
$$

are valid for any fixed $k_{1} \in \mathbb{N}$.

Proof. For (54), we refer to [28], p. 506. Let us prove (55). Using the recurrence formula $J_{k_{1}}^{\prime}(z)=\frac{1}{2}\left(J_{k_{1}-1}(z)-J_{k_{1}+1}(z)\right)$ (see Reference [28], p. 45) and the asymptotic relation $J_{k_{1}}(z)=\sqrt{\frac{2}{\pi z}}\left[\cos \left(z-\frac{k_{1} \pi}{2}-\frac{\pi}{4}\right)+O\left(\frac{1}{z}\right)\right]$ as $z \rightarrow+\infty$ (see Reference [28], p. 199), we deduce

$$
J_{k_{1}}^{\prime}(z)=-\sqrt{\frac{2}{\pi z}}\left[\sin \left(z-\frac{k_{1} \pi}{2}-\frac{\pi}{4}\right)+O\left(\frac{1}{z}\right)\right] \text { as } z \rightarrow+\infty .
$$

Thus, taking (54) into account, we obtain

$$
\begin{aligned}
& \left.\frac{d}{d r} J_{k_{1}}\left(z_{k_{1}, k_{2}} r\right)\right|_{r=1}=\left.J_{k_{1}}^{\prime}\left(z_{k_{1}, k_{2}} r\right)\right|_{r=1} z_{k_{1}, k_{2}} \\
& =-z_{k_{1}, k_{2}} \sqrt{\frac{2}{\pi z_{k_{1}, k_{2}}}}\left[\sin \left(z_{k_{1}, k_{2}}-\frac{k_{1} \pi}{2}-\frac{\pi}{4}\right)+O\left(\frac{1}{k_{2}}\right)\right] \\
& =-\sqrt{\frac{2 z_{k_{1}, k_{2}}}{\pi}}\left[\sin \left(k_{2} \pi-\frac{\pi}{2}+\epsilon_{k_{2}}\right)+O\left(\frac{1}{k_{2}}\right)\right] \text { as } k_{2} \rightarrow \infty,
\end{aligned}
$$

where $\epsilon_{k_{2}}=O\left(\frac{1}{k_{2}}\right)$. Applying the mean value theorem we have

$$
\sin \left(k_{2} \pi-\frac{\pi}{2}+\epsilon_{k_{2}}\right)=\sin \left(k_{2} \pi-\frac{\pi}{2}\right)+\cos \left(\xi_{k_{2}}\right) \epsilon_{k_{2}}=(-1)^{k_{2}-1}+O\left(\frac{1}{k_{2}}\right),
$$

where $\xi_{k_{2}}$ lies between $k_{2} \pi-\frac{\pi}{2}$ and $k_{2} \pi-\frac{\pi}{2}+\epsilon_{k_{2}}$. Using this in (56), we obtain (55).

Theorem 3. Let $\alpha \in(0,1) \cup(1,2)$ and $\omega=\left\{x \in \mathbb{R}^{2}: x_{1}^{2}+x_{2}^{2}<1\right\}$. Then, the statement of Theorem 2 is valid. 
Proof. Let us define $h:=\left.u\right|_{(0, T) \times \partial \omega \times(0,1)}, g_{0}:=\left.u\right|_{(0, T) \times \omega \times\{0\}}, g_{1}:=\left.u\right|_{(0, T) \times \omega \times\{1\}}$. Then, in view of Proposition 1, we have

$$
\begin{aligned}
& u_{k, l}(t)=e_{\alpha}\left(-\lambda_{k, l} ; t\right) *\left(-\int_{\partial \omega} \partial_{\nu} v_{k}(x) h_{l}(t, x) d S\right. \\
& \left.+\pi l g_{0 ; k}(t)-(-1)^{l} \pi l g_{1 ; k}(t)\right), \quad t \in[0, T], \quad k \in \mathcal{K}, l \in \mathbb{N} .
\end{aligned}
$$

By (53), we have

$$
\begin{aligned}
& \partial_{v} v_{k}(x)=\left.\frac{d}{d r} J_{k_{1}}\left(z_{k_{1}, k_{2}} r\right)\right|_{r=1} \cos \left(k_{1} \theta\right), \quad k=\left(k_{1}, k_{2}, 1\right), \\
& \partial_{\nu} v_{k}(x)=\left.\frac{d}{d r} J_{k_{1}}\left(z_{k_{1}, k_{2}} r\right)\right|_{r=1} \sin \left(k_{1} \theta\right), \quad k=\left(k_{1}, k_{2}, 2\right)
\end{aligned}
$$

for $x=(\cos \theta, \sin \theta), \theta \in(-\pi, \pi]$. Let us define

$$
\begin{aligned}
& \widehat{h}_{l}(t, \theta)=h_{l}(t, \cos \theta, \sin \theta), \theta \in(-\pi, \pi], \\
& h_{k_{1}, 1, l}(t)=\int_{-\pi}^{\pi} \widehat{h}_{l}(t, \theta) \cos \left(k_{1} \theta\right) d \theta, \quad h_{k_{1}, 2, l}(t)=\int_{-\pi}^{\pi} \widehat{h}_{l}(t, \theta) \sin \left(k_{1} \theta\right) d \theta .
\end{aligned}
$$

Then, we obtain

$$
\begin{aligned}
& \int_{\partial \omega} \partial_{\nu} v_{k}(x) h_{l}(t, x) d S=\left.\frac{d}{d r} J_{k_{1}}\left(z_{k_{1}, k_{2}} r\right)\right|_{r=1} h_{k_{1}, \sigma, l}(t), \\
& k=\left(k_{1}, k_{2}, \sigma\right) \in \mathbb{N}^{2} \times\{1 ; 2\}, l \in \mathbb{N} .
\end{aligned}
$$

Thus, from (57), we have

$$
\begin{aligned}
& u_{k, l}(t)=e_{\alpha}\left(-\lambda_{k, l} ; t\right) *\left(-\left.\frac{d}{d r} J_{k_{1}}\left(z_{k_{1}, k_{2}} r\right)\right|_{r=1} h_{k_{1}, \sigma, l}(t)\right. \\
& \left.+\pi l g_{0 ; k}(t)-(-1)^{l} \pi l g_{1 ; k}(t)\right), \quad t \in[0, T], \\
& k=\left(k_{1}, k_{2}, \sigma\right) \in \mathbb{N}^{2} \times\{1 ; 2\}, l \in \mathbb{N} .
\end{aligned}
$$

We will show that $h_{k_{1}, \sigma, l}=0, g_{0 ; k}=0, g_{1 ; k}=0$ for $k=\left(k_{1}, k_{2}, \sigma\right) \in \mathbb{N}^{2} \times\{1 ; 2\}, l \in \mathbb{N}$. This proves $u=0$.

Since $h_{k_{1}, \sigma, l}(t)=0, g_{1 ; k}(t)=0$ and $g_{1 ; k}=0$ for $t \in(T-\delta, T), u_{k, l}(T)=0$ and $\lambda_{k, l}=z_{k_{1}, k_{2}}^{2}+\pi^{2} l^{2}+q,(61)$ gives

$$
\begin{aligned}
& 0=-\left.\frac{d}{d r} J_{k_{1}}\left(z_{k_{1}, k_{2}} r\right)\right|_{r=1} \int_{0}^{T-\delta} e_{\alpha}\left(z_{k_{1}, k_{2}}^{2}-\pi^{2} l^{2}-q ; T-\tau\right) h_{k_{1}, \sigma, l}(\tau) d \tau \\
& +\pi l \int_{0}^{T-\delta} e_{\alpha}\left(-z_{k_{1}, k_{2}}^{2}-\pi^{2} l^{2}-q ; T-\tau\right)\left(g_{0 ; k}(\tau)-(-1)^{l} g_{1 ; k}(\tau)\right) d \tau, \\
& k=\left(k_{1}, k_{2}, \sigma\right) \in \mathbb{N}^{2} \times\{1 ; 2\}, l \in \mathbb{N} .
\end{aligned}
$$

Let us choose some $N \in \mathbb{N}$. Since the families $h_{k_{1}, \sigma, l}$ and $g_{0 ; k}-(-1)^{l} g_{1 ; k}$ are bounded in $L_{1}(0, T-\delta)$ and $z_{k_{1}, k_{2}} \rightarrow \infty$ as $k_{2} \rightarrow \infty$ (see (54)), Lemma 3 with $K=\left(k_{2}, l\right)$ implies the relation

$$
\begin{aligned}
& \sum_{n=1}^{N}\left[b_{k_{1}, \sigma, l ; n} \frac{\left.\frac{d}{d r} J_{k_{1}}\left(z_{k_{1}, k_{2}} r\right)\right|_{r=1}}{\left(z_{k_{1}, k_{2}}+\pi^{2} l^{2}+q\right)^{n+1}}+c_{k_{1}, k_{2}, \sigma, l ; n} \frac{\pi l}{\left(z_{k_{1}, k_{2}}^{2}+\pi^{2} l^{2}+q\right)^{n+1}}\right] \\
& =\left.\frac{d}{d r} J_{k_{1}}\left(z_{k_{1}, k_{2}} r\right)\right|_{r=1} O\left(\frac{1}{\left(z_{k_{1}, k_{2}}^{2}+\pi^{2} l^{2}+q\right)^{N+2}}\right)+l O\left(\frac{1}{\left(z_{k_{1}, k_{2}}^{2}+\pi^{2} l^{2}+q\right)^{N+2}}\right) \\
& \text { as } k_{2}^{2}+l^{2} \rightarrow \infty
\end{aligned}
$$


for any fixed $k_{1} \in \mathbb{N}$ and $\sigma \in\{1 ; 2\}$, where

$$
\begin{aligned}
& b_{k_{1}, \sigma, l ; n}=-\frac{(-1)^{n}}{\Gamma(-n \alpha)} \int_{0}^{T-\delta} \frac{h_{k_{1}, \sigma, l}(\tau) d \tau}{(T-\tau)^{n \alpha+1}}, \\
& c_{k_{1}, k_{2}, \sigma, l ; n}=\frac{(-1)^{n}}{\Gamma(-n \alpha)} \int_{0}^{T-\delta} \frac{\left(g_{0 ; k}(\tau)-(-1)^{l} g_{1 ; k}(\tau)\right) d \tau}{(T-\tau)^{n \alpha+1}} .
\end{aligned}
$$

With the help of Lemma 6, we transform (63) to

$$
\begin{aligned}
& \sum_{n=1}^{N}\left[b_{k_{1}, \sigma, l ; n} \frac{\left.\frac{d}{d r} J_{k_{1}}\left(z_{k_{1}, k_{2}} r\right)\right|_{r=1}}{\left(z_{k_{1}, k_{2}}^{2}+\pi^{2} l^{2}+q\right)^{n+1}}+c_{k_{1}, k_{2}, \sigma, l ; n} \frac{\pi l}{\left(z_{k_{1}, k_{2}}^{2}+\pi^{2} l^{2}+q\right)^{n+1}}\right] \\
& =O\left(\frac{1}{\left(z_{k_{1}, k_{2}}^{2}+\pi^{2} l^{2}+q\right)^{N+3 / 2}}\right) \text { as } k_{2}^{2}+l^{2} \rightarrow \infty
\end{aligned}
$$

for any fixed $k_{1} \in \mathbb{N}$ and $\sigma \in\{1 ; 2\}$.

As in previous proofs, we use the induction. We will show that the following equalities are valid for $n=1, \ldots, N$ :

$$
\left.\begin{array}{l}
\frac{1}{\Gamma(-n \alpha)} \int_{0}^{T-\delta} \frac{h_{k_{1}, \sigma, l}(\tau) d \tau}{(T-\tau)^{n \alpha+1}}=0 \\
\frac{1}{\Gamma(-n \alpha)} \int_{0}^{T-\delta} \frac{g_{0 ; k}(\tau) d \tau}{(T-\tau)^{n \alpha+1}}=0, \frac{1}{\Gamma(-n \alpha)} \int_{0}^{T-\delta} \frac{g_{1 ; k}(\tau) d \tau}{(T-\tau)^{n \alpha+1}}=0 \\
k=\left(k_{1}, k_{2}, \sigma\right) \in \mathbb{N}^{2} \times\{1 ; 2\}, l \in \mathbb{N} .
\end{array}\right\} .
$$

Suppose that either $\hat{n}=1$ or $\hat{n} \in\{2 ; \ldots ; N\}$ and (65) are valid for $n=1, \ldots, \hat{n}-1$. We have to to show that, then, (65) holds for $n=\hat{n}$, too.

Due to the supposition from (63) we have

$$
\begin{aligned}
& \sum_{n=\hat{n}}^{N}\left[b_{k_{1}, \sigma, l ; n} \frac{\left.\frac{d}{d r} J_{k_{1}}\left(z_{k_{1}, k_{2}} r\right)\right|_{r=1}}{\left(z_{k_{1}, k_{2}}^{2}+\pi^{2} l^{2}+q\right)^{n+1}}+c_{k_{1}, k_{2}, \sigma, l ; n} \frac{\pi l}{\left(z_{k_{1}, k_{2}}^{2}+\pi^{2} l^{2}+q\right)^{n+1}}\right] \\
& =O\left(\frac{1}{\left(z_{k_{1}, k_{2}}^{2}+\pi^{2} l^{2}+q\right)^{N+3 / 2}}\right) \text { as } k_{2}^{2}+l^{2} \rightarrow \infty
\end{aligned}
$$

for any fixed $k_{1} \in \mathbb{N}$ and $\sigma \in\{1 ; 2\}$. Multiplying (66) by $\frac{\left(z_{k_{1}, k_{2}}^{2}+\pi^{2} l^{2}+q\right)^{\hat{n}+1}}{\left.\frac{d}{d r} r_{k_{1}}\left(z_{k_{1}, k_{2}} r\right)\right|_{r=1}}$ and taking Lemma 6 into account, we obtain $b_{k_{1}, \sigma, l ; n}=O\left(\frac{1}{\sqrt{k_{2}}}\right)$ as $k_{2} \rightarrow \infty$ for any fixed $l \in \mathbb{N}, k_{1} \in \mathbb{N}$ and $\sigma \in\{1 ; 2\}$. Passing to the limit $k_{2} \rightarrow \infty$, we have $b_{k_{1}, \sigma, l ; n}=0$ for any $l \in \mathbb{N}, k_{1} \in \mathbb{N}$ and $\sigma \in\{1 ; 2\}$. Therefore, the first equality in (65) holds for $n=\hat{n}$. Next, let us multiply (66) by $\frac{\left(z_{k_{1}, k_{2}}^{2}+\pi^{2} l^{2}+q\right)^{\hat{n}+1}}{\pi l}$. Then, we deduce $c_{k_{1}, k_{2}, \sigma, l ; \hat{n}}=O\left(\frac{1}{l}\right)$ as $l \rightarrow \infty$ for any fixed $k_{1}, k_{2} \in \mathbb{N}$ and $\sigma \in\{1 ; 2\}$. Therefore,

$$
\frac{1}{\Gamma(-\hat{n} \alpha)} \int_{0}^{T-\delta} \frac{\left(g_{0 ; k}(\tau)-(-1)^{l} g_{1 ; k}(\tau)\right) d \tau}{(T-\tau)^{\hat{n} \alpha+1}}=O\left(\frac{1}{l}\right) \text { as } l \rightarrow \infty
$$

for any fixed $k_{1}, k_{2} \in \mathbb{N}$ and $\sigma \in\{1 ; 2\}$. Passing to the limit $l \rightarrow \infty$ separately for even and odd $l$, we obtain

$$
\frac{1}{\Gamma(-\hat{n} \alpha)} \int_{0}^{T-\delta} \frac{\left(g_{0 ; k}(\tau) \mp g_{1 ; k}(\tau)\right) d \tau}{(T-\tau)^{\hat{n} \alpha+1}}=0
$$


for any $k=\left(k_{1}, k_{2}, \sigma\right) \in \mathbb{N}^{2} \times\{1 ; 2\}$. This implies the second and third equalities in (65) for $n=\hat{n}$. The induction is complete.

We have proven that (65) is valid in case $n \in\{1 ; \ldots ; N\}$. Since $N \in \mathbb{N}$ is arbitrary, (65) is valid for all $n \in \mathbb{N}$. Lemma 4 implies the desired relations $h_{k_{1}, \sigma, l}=0, g_{0 ; k}=0, g_{1 ; k}=0$, $k=\left(k_{1}, k_{2}, \sigma\right) \in \mathbb{N}^{2} \times\{1 ; 2\}, l \in \mathbb{N}$. The theorem is proved.

At the end of this subsection, we establish the uniqueness of a solution of an inverse problem on the disk $\omega=\left\{x \in \mathbb{R}^{2}: x_{1}^{2}+x_{2}^{2}<1\right\}$. This problem requires to find a function $u(t, x)$ that satisfies the equation $D_{t}^{\alpha-\lfloor\alpha\rfloor}\left[\partial_{t}^{\lfloor\alpha\rfloor} u-\varphi_{\lfloor\alpha\rfloor}\right](t, x)-\left(\partial_{x_{1}}^{2}+\partial_{x_{2}}^{2}-q\right) u(t, x)=F(t, x)$ for $t \in(0, T), x \in \omega$, the initial condition(s) $\left.\partial_{t}^{j} u(t, x)\right|_{t=0}=\varphi_{j}(x)$ for $x \in \omega, j \in\{0 ;\lfloor\alpha\rfloor\}$, the partly given boundary condition $u(t, x)=h(t, x)$ for $t \in(T-\delta, T), x \in \partial \omega$, and the final condition $u(T, x)=\psi(x)$ for $x \in \omega$.

Corollary 2. Let $\alpha \in(0,1) \cup(1,2), \omega=\left\{x \in \mathbb{R}^{2}: x_{1}^{2}+x_{2}^{2}<1\right\}, u \in C^{\lfloor\alpha\rfloor}\left([0, T] ; L_{2}(\omega)\right)$ and $\exists p \in(1, \infty): u \in L_{p}\left((0, T) ; H^{2}(\omega)\right), \partial_{t}^{\lfloor\alpha\rfloor} u \in{ }_{0} H_{p}^{\alpha-\lfloor\alpha\rfloor}\left((0, T) ; L_{2}(\omega)\right)$. Assume that

$$
\begin{aligned}
& D_{t}^{\alpha-\lfloor\alpha\rfloor} \partial_{t}^{\lfloor\alpha\rfloor} u(t, x)-\left(\partial_{x_{1}}^{2}+\partial_{x_{2}}^{2}-q\right) u(t, x)=0, \quad t \in(0, T), \quad x \in \omega, \\
& \left.\partial_{t}^{j} u(t, x)\right|_{t=0}=0, \quad x \in \omega, \quad j \in\{0 ;\lfloor\alpha\rfloor\}, \\
& u(t, x)=0, \quad t \in(T-\delta, T), \quad x \in \partial \omega
\end{aligned}
$$

for some $\delta \in(0, T)$ and

$$
u(T, x)=0, \quad x \in \omega
$$

Then, $u=0$.

Proof. Let us define $\widehat{u}(t, x, y)=u(t, x) \sin (\pi y), t \in[0, T],(x, y) \in \bar{\Omega}$, where $\Omega=\omega \times(0,1)$. Then, $\widehat{u} \in \mathcal{U}_{\alpha}$,

$$
\begin{aligned}
& D_{t}^{\alpha-\lfloor\alpha\rfloor} \partial_{t}^{\lfloor\alpha\rfloor} \widehat{u}(t, x, y)-(\Delta-\widehat{q}) \widehat{u}(t, x, y)=0, t \in(0, T), \quad(x, y) \in \Omega, \\
& \left.\partial_{t}^{j} \widehat{u}(t, x, y)\right|_{t=0}=0, \quad(x, y) \in \Omega, \quad j \in\{0 ;\lfloor\alpha\rfloor\}, \\
& \widehat{u}(t, x, y)=0, \quad t \in(T-\delta, T), \quad x \in \partial \omega, y \in(0,1), \\
& \widehat{u}(t, x, 0)=0, \quad \widehat{u}(t, x, 1)=0, \quad t \in(0, T), x \in \omega, \\
& \widehat{u}(T, x, y)=0, \quad(x, y) \in \Omega,
\end{aligned}
$$

where $\widehat{q}=q-\pi^{2}$. Theorem 3 yields $\widehat{u}=0$. This implies $u=0$.

\section{Severe Ill-Posedness of the Inverse Problems}

IP1 and IP2 are severely ill-posed. This means that a necessary condition for the existence of solutions is an infinite differentiability of certain combinations of the data.

Let us demonstrate this feature for IP1 in the particular case $\varphi_{j}=0, j \in\{0 ;\lfloor\alpha\rfloor\}$, $F=0, h=0$ and $B_{0}=B_{1}=I$. Then, by Proposition 1, Lemma 3, the assumptions $g_{0}(x, t)=g_{1}(x, t)=0, t \in(T-\delta, T), x \in \omega$, and the relation $\varrho_{l}=(\pi l)^{2}$, the Fourier coefficients of the final observation function $\psi(x, y)=u(T, x, y)$ are expanded as

$$
\psi_{k, l}=\sum_{n=1}^{N}\left[c_{k ; n}-(-1)^{l} d_{k ; n}\right] \frac{\pi l}{\left(\mu_{k}+\varrho_{l}\right)^{n}}+O\left(\frac{1}{\varrho_{l}^{N+1 / 2}}\right) \quad \text { as } l \rightarrow \infty
$$

for any fixed $k \in \mathcal{K}$ and $N \in \mathbb{N}$, where

$$
c_{k ; n}=\frac{(-1)^{n}}{\Gamma(-n \alpha)} \int_{0}^{T-\delta} \frac{g_{0 ; k}(\tau) d \tau}{(T-\tau)^{n \alpha+1}}, \quad d_{k ; n}=\frac{(-1)^{n}}{\Gamma(-n \alpha)} \int_{0}^{T-\delta} \frac{g_{1 ; k}(\tau) d \tau}{(T-\tau)^{n \alpha+1}} .
$$


Let us show that the following functions,

$$
\psi_{k}(y)=\left\langle\psi(\cdot, y), v_{k}\right\rangle_{L_{2}(\omega)}, y \in(0,1), \quad k \in \mathbb{N},
$$

are infinitely differentiable in $(0,1)$.

Let us choose some $k \in \mathcal{K}, N \in \mathbb{N}$ and define

$$
p_{k ; 1}(y)=\operatorname{ch}\left(\sqrt{\mu_{k}} y\right)-\frac{\operatorname{ch}\left(\sqrt{\mu_{k}}\right) \operatorname{sh}\left(\sqrt{\mu_{k}} y\right)}{\operatorname{sh} \sqrt{\mu_{k}}}, r_{k ; 1}(y)=\frac{\operatorname{sh}\left(\sqrt{\mu_{k}} y\right)}{\operatorname{sh} \sqrt{\mu_{k}}} .
$$

Then, we have

$$
\left\langle p_{k ; 1}, w_{l}\right\rangle_{L_{2}(0,1)}=\frac{\pi l}{\mu_{k}+\varrho_{l}},\left\langle r_{k ; 1}, w_{l}\right\rangle_{L_{2}(0,1)}=-(-1)^{l} \frac{\pi l}{\mu_{k}+\varrho_{l}} .
$$

Moreover, let functions $p_{k ; n}, r_{k ; n}, n=2,3, \ldots$, be the solutions of the following recursive families of boundary value problems:

$$
\begin{aligned}
& \left(-\frac{d^{2}}{d y^{2}}+\mu_{k}\right) p_{k ; n}(y)=p_{k ; n-1}(y), y \in(0,1), \quad p_{k ; n}(0)=p_{k ; n}(1)=0, \\
& \left(-\frac{d^{2}}{d y^{2}}+\mu_{k}\right) r_{k ; n}(y)=r_{k ; n-1}(y), y \in(0,1), \quad r_{k ; n}(0)=r_{k ; n}(1)=0 .
\end{aligned}
$$

Then, for the function $Z_{k ; N}(y)=\sum_{n=1}^{N}\left[c_{k ; n} p_{k ; n}(y)+d_{k ; n} r_{k ; n}(y)\right]$, the relation

$$
Z_{k, l ; N}=\left\langle Z_{k ; N}, w_{l}\right\rangle_{L_{2}(0,1)}=\sum_{n=1}^{N}\left[c_{k ; n}-(-1)^{l} d_{k ; n}\right] \frac{\pi l}{\left(\mu_{k}+\varrho_{l}\right)^{n}}, \quad l \in \mathbb{N},
$$

is valid. Comparing this with (67), we see $\psi_{k, l}-Z_{k, l ; N}=O\left(\frac{1}{e_{l}^{N+1 / 2}}\right)$ as $l \rightarrow \infty$. Such a decay rate of Fourier coefficients is sufficient to guarantee that the function $\psi_{k}-Z_{k ; N}$ belongs to $H^{2 N}(0,1)$ (cf. Reference [29], Theorem 3.1). Evidently, $Z_{k ; N} \in C^{\infty}(0,1)$. Thus, $\psi_{k} \in H^{2 N}(0,1)$. Since $N \in \mathbb{N}$ is arbitrary, we have $\psi_{k} \in C^{\infty}(0,1)$. That is what we had to show.

\section{Conclusions and Additional Remarks}

We have proven the uniqueness for inverse problems to reconstruct solutions of the fractional diffusion-wave equation in a cylinder $\omega \times(0,1)$ in two cases:

(1) the initial and final data and full boundary data on lateral hypersurface of the cylinder are given, but the boundary data on the bases of the cylinder are specified in a partial time interval $(T-\delta, T)(\mathrm{IP} 1)$;

(2) the initial and final data are given and Dirichlet boundary data on the whole boundary of the cylinder are specified in $(T-\delta, T)$ (IP2). In IP2, we were limited to a cube and a circular cylinder.

We hope that the uniqueness results regarding IP2 can be extended to other cylinders of simple geometry, e.g., cylinders-based annuli, balls, and also to Neumann and Robin boundary conditions. However, the extension to the case of an arbitrary base $\omega$ seems difficult. The reason is that the method applied in Section 5 requires a decomposition of eigenfunctions $v_{k}$ into two factors, one of which forms a complete system in $L_{2}(\partial \omega)$, while the other enables the use of Lemmas 3 and 4 . For instance, in the case of the circular cylinder (Section 5.2), the first factor is either $\cos \left(k_{1} \theta\right)$ or $\sin \left(k_{1} \theta\right)$, and the second one is $J_{k_{1}}\left(z_{k_{1} k_{2}} r\right)$; in case of the cube (Section 5.1), these factors are $\prod_{\substack{j=1 \\ j \neq s}}^{d} \sin \left(k_{j} \pi x_{j}\right)$ and $\sin \left(k_{s} \pi x_{j}\right)$, respectively, where $s$ is an index of a pair of parallel faces of the cube. The aforementioned decomposition is not possible in the case of a general $\omega$. 
IP1 and IP2 are linear, and the uniqueness is valid for solutions that belong to the space $\mathcal{U}_{\alpha}$ defined in (12). The corresponding regularity conditions for the initial data, the source term and the boundary data are given in (13) and (14).

The proofs of uniqueness for IP1 and IP2 work only in case the fractional order $\alpha$ belongs to the set $(0,1) \cup(1,2)$. In the limiting cases $\alpha=1$ and $\alpha=2$, the Mittag-Leffler function $E_{\alpha, \alpha}$ loses the power-type asymptotics, and the basic Lemma 3 fails.

On the other hand, in the literature, one can find several treatments of inverse problems with unknown boundary conditions for the usual diffusion equation that use additional data that are different from the final data. A well-known problem is the Cauchy problem, where the over-specified data are given on a portion of the boundary and nothing is given on the remaining part of the boundary. The uniqueness can be proved by means of Carleman estimates [13].

An open question is the uniqueness for IP1 and IP2 in the case $\delta=0$. Our theory does not work in such a case. The basic Lemma 3 fails because the integrals at the right-hand side of (21) are singular.

Funding: The research was supported by Estonian Research Council Grant nr PRG832.

Data Availability Statement: Not applicable.

Conflicts of Interest: The author declares no conflict of interests.

\section{References}

1. Pskhu, A.V. Green functions of the first boundary-value problem for a fractional diffusion-wave equation in multidimensional domains. Mathematics 2020, 8, 464. [CrossRef]

2. Sakamoto, K.; Yamamoto, M. Initial value/boundary value problems for fractional diffusion-wave equations and applications to some inverse problems. J. Math. Anal. Appl. 2011, 382, 426-447. [CrossRef]

3. Metzler, R.; Klafter, J. The random walk's guide to anomalous diffusion: a fractional dynamics approach. Phys. Rep. 2000, 339, 1-77. [CrossRef]

4. Baeumer, B.; Kurita, S.; Meerschaert, M.M. Inhomogeneous fractional diffusion equations. Fract. Calc. Appl. Anal. 2005, 8, 371-386.

5. Chechkin, A; Gorenflo, R; Sokolov, I. Fractional diffusion in inhomogeneous media. J. Phys. A Math. Gen. 2005, 38, 679-684. [CrossRef]

6. Luchko, Y. Fractional wave equation and damped waves. J. Math. Phys. 2013, 54, 031505. [CrossRef]

7. Mainardi, F. Fractional diffusive waves in viscoelastic solids. In Nonlinear Waves in Solids; Wegner, J.L., Norwood, F.R., Eds.; ASME/AMR: Fairfield, CT, USA, 1995; pp. 93-97.

8. Šš̌kova, K.; Slodička, M. A source identification problem in a time-fractional wave equation with a dynamical boundary condition. Comput. Math. Appl. 2018, 75, 4337-4354. [CrossRef]

9. Ismailov, M.I.; Çiçek, M. Inverse source problem for a time-fractional diffusion equation with nonlocal boundary conditions. Appl. Math. Model. 2016, 40, 4891-4899. [CrossRef]

10. Kian, Y.; Yamamoto, M. Reconstruction and stable recovery of source terms and coefficients appearing in diffusion equations. Inverse Probl. 2019, 35, 115006. [CrossRef]

11. Kian, Y.; Soccorsi, E.; Xue, Q.; Yamamoto, M. Identification of time-varying source term in time-fractional evolution equations. arXiv 2021, arXiv:1911.09951. to appear in Fract. Calc. Appl. Anal.

12. Lopushanska, H.; Lopushansky, A.; Myaus, O. Inverse problems of periodic spatial distributions for a time fractional diffusion equation. Electron. J. Differ. Equ. 2016, 2016, 1-9.

13. Isakov, V. Inverse Problems for Partial Differential Equations, 2nd ed.; Springer: New York, NY, USA, 2006.

14. Jin, B.; Rundell, W. A tutorial on inverse problems for anomalous diffusion processes. Inverse Probl. 2015, 31, 035003. [CrossRef]

15. Kinash, N.; Janno, J. Inverse problems for a generalized subdiffusion equation with final overdetermination. Math. Model. Anal. 2019, 24, 236-262.

16. Kirane, M.; Samet, B.; Torebek, B. Determination of an unknown source term temperature distribution for the sub-diffusion equation at the initial and final data. Electron. J. Differ. Equ. 2017, 2017, 1-13.

17. Janno, J.; Kasemets, K. Uniqueness for an inverse problem for a semilinear time fractional diffusion equation. Inverse Probl. Imaging 2017, 11, 125-149. [CrossRef]

18. Ali, M.;Aziz, S.; Malik, S.A. Inverse problem for a space-time fractional diffusion equation: Application of fractional SturmLiouville operator. Math. Meth. Appl. Sci. 2018, 41, 2733-2747. [CrossRef]

19. Janno, J.; Kinash, N. Reconstruction of an order of derivative and a source term in a fractional diffusion equation from final measurements. Inverse Probl. 2018, 34, 025007. [CrossRef] 
20. Orlovsky, D. Parameter determination in a differential equation of fractional order with Riemann-Liouville fractional derivative in a Hilbert space. J. Sib. Fed. Univ. Math. Phys. 2015, 8, 55-63. [CrossRef]

21. Yamamoto, M.; Zhang, Y. Conditional stability in determining a zeroth-order coefficient in a half-order fractional diffusion equation by a Carleman estimate. Inverse Probl. 2012, 28, 105010. [CrossRef]

22. Janno, J. Determination of time-dependent sources and parameters of nonlocal diffusion and wave equations from final data. Fract. Calc. Appl. Anal. 2020, 23, 1678-1701. [CrossRef]

23. Ern, A.; Guermond, J.-L. Theory and Practice of Finite Elements; Springer: New York, NY, USA, 2004.

24. Zacher, R. Quasilinear Parabolic Problems with Nonlinear Boundary Conditions. Ph.D. Dissertation, Martin-Luther-Universität Halle-Wittenberg, Halle, Germany, 2003. Available online: https://www.yumpu.com/en/document/view/4926858/quasilinearparabolic-problems-with-nonlinear-boundary-conditions (accessed on 2003).

25. Samko, S.G.; Kilbas, A.A.; Marichev, O.I. Fractional Integrals and Derivatives. Theory and Applications; Gordon and Breach Science Publishers: Amsterdam, The Netherlands, 1993.

26. Gorenflo, R.; Kilbas, A.A.; Mainardi, F.; Rogosin, S.V. Mittag-Leffler Functions, Related Topics and Applications; Springer: New York, NY, USA, 2014.

27. Kilbas, A.A.; Srivastava, H.M.; Trujillo, J.J. Theory and Applications of Fractional Differential Equations; Elsevier: Amsterdam, The Netherlands, 2006.

28. Watson, N.G. A Treatise on the Theory of Bessel Functions; Cambridge University Press: Cambridge, UK, 1966.

29. Nissilä, J. Fourier decay of absolutely and Hölder continuous functions with infinitely or finitely many oscillations. arXiv 2018, arXiv:1805.02445. 